\title{
Programmable large DNA deletion, replacement, integration, and inversion with twin prime editing and site-specific recombinases
}

Andrew V. Anzalone*, Xin D. Gao*, Christopher J. Podracky*, Andrew T. Nelson, Luke W. Koblan, Aditya Raguram, Jonathan M. Levy, Jaron A. M. Mercer and David R. Liu1,2,3ł

${ }^{1}$ Merkin Institute of Transformative Technologies in Healthcare, Broad Institute of Harvard and MIT, Cambridge, Massachusetts, USA

${ }^{2}$ Department of Chemistry and Chemical Biology, Harvard University, Cambridge, Massachusetts, USA

${ }^{3}$ Howard Hughes Medical Institute, Harvard University, Cambridge, Massachusetts, USA

* These authors contributed equally

‡ Correspondence should be addressed to David R. Liu: drliu@fas.harvard.edu 


\section{Summary}

The targeted deletion, replacement, integration, or inversion of DNA sequences at specified locations in the genome could be used to study or treat many human genetic diseases. Here, we describe twin prime editing (twinPE), a method for the programmable replacement or excision of DNA sequence at endogenous human genomic sites without requiring double-strand DNA breaks. TwinPE uses a prime editor $(\mathrm{PE})$ protein and two prime editing guide RNAs (pegRNAs) that template the synthesis of complementary DNA flaps on opposing strands of genomic DNA, resulting in the replacement of endogenous DNA sequence between the PE-induced nick sites with pegRNA-encoded sequences. We show that twinPE in human cells can perform precise deletions of at least $780 \mathrm{bp}$ and precise replacements of genomic DNA sequence with new sequences of at least $108 \mathrm{bp}$. By combining single or multiplexed twinPE with site-specific serine recombinases either in separate steps or in a single step, we demonstrate targeted integration of gene-sized DNA plasmids (>5,000 bp) into safe-harbor loci including AAVS1, CCR5, and ALB in human cells. To our knowledge, these results represent the first RNA-programmable insertion of genesized DNA sequences into targeted genomic sites of unmodified human cells without requiring double-strand breaks or homology-directed repair. Twin PE combined with recombinases also mediated a 40,167-bp inversion at IDS that corrects a common Hunter syndrome allele. TwinPE expands the capabilities of precision gene editing without requiring double-strand DNA breaks and synergizes with other tools to enable the correction or complementation of large or complex pathogenic alleles in human cells. 
Disease-associated human genetic variants arise through a variety of sequence changes, ranging from single-base pair substitutions to mega base-scale duplications, deletions and rearrangements ${ }^{1-3}$. Gene editing approaches that can install, correct, or complement these pathogenic variants in human cells have the potential to advance our understanding of human genetic disease and could also lead to the development of new therapeutics ${ }^{4,5}$. Several mammalian cell gene editing approaches based on CRISPR-Cas systems have been developed over the past decade ${ }^{6}$, including nucleases ${ }^{7-9}$, base editors $^{10,11}$, and prime editors ${ }^{12}$, each of which has the potential to address a subset of known pathogenic sequence changes.

CRISPR-Cas nucleases such as Cas9 can be used to disrupt genes through the generation of double-strand DNA breaks (DSBs) that lead to stochastic indels. In addition, paired Cas9 nuclease strategies have been developed for the targeted deletion of genomic DNA sequences ranging from $\sim 50$ to $>100,000$ base pairs in length ${ }^{13}$. By providing a linear donor DNA sequence, targeted insertion of new DNA sequences can be performed at single cut sites or between paired cut sites through end-joining or homology-directed repair (HDR) processes $^{14,15}$.

While versatile in some ways, single-nuclease and paired-nuclease editing approaches have substantial drawbacks. HDR is inefficient compared to the generation of uncontrolled mixtures of indels in the vast majority of cell types ${ }^{16,17}$. Likewise, the use of paired nucleases for targeted deletion also generates multiple byproducts, including the desired deletion accompanied by undesired indels, indels at individual DSB sites without the desired deletion, undesired inversion of the intervening sequence between DSB sites, and unintended integration of exogenous DNA sequence at DSB sites ${ }^{13,18}$. In addition, the precise location of the deletions is restricted by PAM availability, which dictates the location of nuclease-mediated DNA cutting. Similar restrictions and byproducts exist for DNA donor knock-in, which typically occurs without control of sequence orientation when homologyindependent approaches are used ${ }^{14}$ and is accompanied by efficient indel byproduct generation ${ }^{19}$. Finally, the simultaneous generation of multiple DSBs at on-target or off-target sites can promote large deletions ${ }^{20-22}$, chromosomal rearrangements such as translocations $^{23}$, and chromothripsis ${ }^{24}$. The tendency of DSBs to generate large mixtures of undesired byproducts and chromosomal changes ${ }^{25-27}$ poses considerable challenges when applying nuclease-based editing strategies for the excision, replacement, or insertion of larger DNA sequences, especially in therapeutic settings. 
Base editors can be used to precisely install $\mathrm{C} \cdot \mathrm{G}-\mathrm{to}-\mathrm{T} \cdot \mathrm{A}^{10}$ or $\mathrm{A} \cdot \mathrm{T}$-to-G•C $\mathrm{C}^{11}$ transition mutations, or $\mathrm{C} \cdot \mathrm{G}-\mathrm{to}-\mathrm{G} \cdot \mathrm{C}$ transversion mutations ${ }^{28-30}$ without requiring $\mathrm{DSBs}{ }^{6}$. Prime editors can precisely install any of the twelve possible base pair substitutions as well as small insertions and deletions without requiring $\mathrm{DSBs}^{12}$. In their original form, however, these DSBfree editing technologies cannot be directly used to efficiently install edits that alter hundreds or thousands of base pairs, such as large deletions, gene-sized insertions, large replacements, or large inversions.

Prime editing has been shown to be capable of making precise insertions of up to $\sim 40$ bp and deletions of up to $~ 80 \mathrm{bp}$ with PE2 and PE3 systems in human cells with high ratios of desired edits to byproducts ${ }^{12}$. While prime editing offers the flexibility to replace one DNA segment with a new sequence, PE2 and PE3 systems have not yet been able to mediate larger insertions and deletions of the size of typical exons or gene coding sequences, and the presumed mechanism of simple prime editing reactions makes these larger DNA changes difficult by requiring long pegRNA reverse transcription templates and long-range DNA polymerization. In contrast, site-specific DNA recombinases have the ability to perform DNA excision, inversion, integration, or exchange of large DNA sequences in mammalian cells ${ }^{31,32}$. The longstanding challenge of reprogramming site-specific recombinases ${ }^{33-36}$, however, has limited their use for precision gene editing applications.

Here, we report the development of twin prime editing (twinPE), which enables the deletion, substitution, or insertion of larger DNA sequences at targeted endogenous genomic sites with high efficiencies in human cells. In addition to these capabilities, twinPE can also be used to integrate one or more recombinase recognition sites with high efficiency at targeted sites in the human genome. These recognition sites can then be used as substrates for site-specific serine recombinases to enable the targeted integration of gene-sized DNA segments, or the targeted inversion of gene-sized genomic DNA sequences. The twinPE and recombinase reagents can be delivered either stepwise, or simultaneously to enable one-step targeted integration or inversion of large DNA segments at endogenous genomic loci.

\section{Results}

\section{Twin prime editing strategy}

Prime editing uses a prime editor protein comprising a fusion of a catalytically impaired Cas9 nickase and a wild-type (PE1) or engineered (PE2) MMLV reverse transcriptase (RT) enzyme, and a prime editing guide RNA (pegRNA) that both specifies the target genomic site 
and encodes the desired edit ${ }^{12}$. Upon target site recognition, PE•pegRNA complexes nick the PAM-containing DNA strand and directly reverse transcribe the pegRNA's RT template into genomic DNA using the nicked strand as a primer. Following reverse transcription, the newly synthesized 3' flap containing the edited sequence invades the adjacent DNA to replace the redundant 5' flap sequence. The opposing nonedited strand is then repaired using the edited DNA strand as a template. This proposed prime editing pathway presents at least two opportunities for cellular DNA repair to reject the desired edit and revert the DNA sequence to its original form: during 3' flap annealing and ligation, and during heteroduplex resolution.

We hypothesized that bypassing potentially disfavored steps in DNA repair might allow prime editing to occur with increased efficiency and enable new classes of targeted gene edits in mammalian cells. We envisioned a twin prime editing (twinPE) strategy that uses a pair of pegRNAs, each of which targets a different DNA strand and templates the synthesis of a 3' flap that is complementary to the 3' flap templated by the other pegRNA (Fig. 1a). We hypothesized that if the newly synthesized DNA strands were highly dissimilar to the endogenous target site, the complementary 3' flaps would preferentially hybridize with each other to create an intermediate containing annealed 3' overhangs of new DNA sequence and annealed 5' overhangs of original DNA sequence (Fig. 1a). As both edited strands are synthesized by prime editor complexes, there is no requirement for invasion of the target site by edit-containing flap strands, or for the edit to be copied to the complementary DNA strand. Excision of the original DNA sequence (annealed 5' overhangs), filling in of gaps by polymerases, and ligation of the pair of nicks would result in the replacement of the endogenous sequence between the nick sites with the paired 3' flap sequences (Fig. 1a). Due to the flexibility in template design, the edit could in principle insert a new DNA sequence, delete a portion of original DNA sequence, or replace a portion of original DNA.

\section{TwinPE-mediated large sequence replacement and deletion}

To test the twinPE strategy, we initially targeted the HEK293T site 3 locus (hereafter referred to as HEK3) in HEK293T cells to replace 90 bp of endogenous sequence with a 38bp Bxb1 recombinase attB substrate sequence ${ }^{37}$ (Fig. 1b). For each protospacer, three pegRNAs were designed with RT templates that contained 30, 34, or $38 \mathrm{nt}$ of the 38-bp attB sequence (Fig. 1c). Pairwise combinations of these pegRNAs are predicted to generate 3' flaps with overlapping complementarity ranging from 22 to $38 \mathrm{bp}$ (Fig. 1c). When both pegRNAs were transfected into HEK293T cells along with PE2, we observed highly efficient 
attB site insertion, with some combinations of pegRNAs yielding $>80 \%$ conversion to the desired product (Fig. 1c). A similar strategy for the replacement of the 90-bp endogenous sequence with the 50-bp Bxb1 attP attachment sequence also achieved high editing efficiencies up to $58 \%$ (Fig. 1c). Notably, it was not necessary for either pegRNA to encode the full insertion sequence, since partially overlapping complementary flaps enabled fulllength attB or attP sequence incorporation. However, 3' flaps with greater overlap led to slightly higher editing efficiencies and fewer indels for insertion of the Bxb1 attB and attP sequences at HEK3 (Fig. 1c).

Independent of our efforts, Shendure and co-workers ${ }^{38}$ and Gao and co-workers ${ }^{39}$ reported elegant dual-pegRNA prime editing systems that can be used to perform precise large deletions (up to $710 \mathrm{bp}$ ) in human cells or improve base substitution and small insertion or deletion edits in plant cells, respectively. For each of these strategies, the templated 3' flap sequence is homologous to the target site DNA sequence to facilitate DNA repair for incorporation of the edit (Fig. 2a). In contrast, twinPE was designed to bypass the need for any homologous DNA sequence in the pegRNA RT template, thus offering more template sequence flexibility and the freedom to mediate larger insertions.

Next, we tested if twinPE could support the insertion of DNA sequences larger than the $\leq 44-$ bp insertions previously demonstrated ${ }^{12}$ using PE2 or PE3 prime editing systems. First, we examined PE3-mediated insertions of FKBP12 coding fragments ranging from 12 to 108 bp. Targeting the HEK3 locus with PE3 in HEK293T cells, we achieved moderate editing efficiencies for the shorter 12-bp and 36-bp insertions (32\% and $17 \%$, respectively), but very little desired product for the 108-bp insertion (0.80\%) (Fig. 2b). By contrast, twinPE enabled $16 \%$ insertion efficiency for the 108-bp fragment with concomitant deletion of 90 bp of HEK3 sequence, a 20-fold improvement over a the 108-bp insertion with PE3. Similarly, twinPE was 2- to 4-fold more efficient than PE3 for inserting a 108-bp FKBP12 cDNA fragment at CCR5 (Extended Data Fig. 1). Finally, 113-bp and 103-bp insertions containing pairs of Bxb1 recombinase sites (attB-27 bp spacer-attP or attB-27 bp spacer-attB) were installed with similar efficiencies (11\% and 9.7\%, respectively) at CCR5 using twinPE (Extended Data Fig. 1). These results demonstrate the ability of twinPE to mediate larger insertions than have been previously demonstrated with PE2 and PE3 systems.

One potential application of the twinPE strategy is the replacement of exonic coding sequence with recoded DNA sequence that maintains the protein sequence and has the potential to correct any combination of mutations between target twinPE-induced nick sites. 
Such a capability raises the possibility of using a single pegRNA set and prime editor to correct any of a variety of pathogenic mutations within a stretch of DNA, potentially addressing multiple cohorts of animal models or patients. To test this approach, we targeted $P A H$, encoding phenylalanine hydroxylase. Mutations within $P A H$ cause the genetic metabolic disorder phenylketonuria $(P K U)^{40}$. We tested the ability of twin $P E$ to recode portions of $P A H$ exon 4 and exon 7, which commonly harbor mutations in PKU patients, in HEK293T cells. By testing different flap overlap lengths using engineered pegRNAs (epegRNAs) containing a 3' evoPreQ1 motif ${ }^{41}$, we achieved the desired sequence recoding with average efficiencies of up to $23 \%$ for a 46 -bp recoding and up to $27 \%$ for a longer overlapping 64-bp recoding in exon 7, and up to 9.4\% average efficiency for a 64-bp recoding of exon 4 (Fig. 2c and Extended Data Fig. 2). Additional exons in PAH could also be recoded, albeit with lower efficiency (Extended Data Fig. 2). These results demonstrate that twinPE can replace stretches of dozens of nucleotides in human cells with a single pair of pegRNAs.

In addition to insertion and replacement of DNA sequences, twinPE may also mediate precise deletions more effectively than previously described methods. Paired-nuclease deletion strategies generate deletions of sequence between the two DSBs and are thus restricted by PAM availability. In addition, desired deletions are accompanied by undesired indel and inversion byproducts. In contrast, twinPE has the potential to generate deletions with greater flexibility and precision due to the lack of required DSBs and the ability to write additional DNA sequence at the twinPE-induced nick sites. To assess twinPE for precise deletions, we compared three strategies using paired pegRNAs: a "single-anchor" (SA) twinPE strategy, which fixes the deletion junction at one of the two nick sites; a "hybridanchor" (HA) twinPE strategy, which allows flexible deletion junctions between the nick sites; and, the PrimeDel (PD) strategy recently reported by Shendure and coworkers ${ }^{38}$, which generates deletions between the nick sites with the option of inserting additional DNA sequence (Fig. 2d). Each strategy differs in the relationship of the sequences of the two flaps and thus in the positioning of the deletion with respect to the nick sites (Fig. $2 \mathrm{~d}$ ).

Targeting the HEK3 site in HEK293T cells and using the single-anchor strategy, 13-nt complementary flaps were used to delete $77 \mathrm{bp}$ of sequence adjacent to one of the pegRNAinduced nick sites with $15 \%$ efficiency and $2.1 \%$ indels (Fig. 2e, SA- $\Delta 77$ ), and 34-nt complementary flaps were used to precisely excise 56 bp of sequence with $19 \%$ efficiency and $4.2 \%$ indels (Fig. 2e, SA- $\Delta 56$ ). Using the hybrid-anchor strategy, we deleted 64 bp between the pegRNA-induced nick sites such that the product retains 13 bp of sequence 3' of 
each nick with $12 \%$ efficiency and $5.5 \%$ indels (Fig. 2e, HA- $\Delta 64$ ). Finally, we tested the PrimeDel strategy for the deletion of $90 \mathrm{bp}$ between the pegRNA-induced nick sites, which occurred with $40 \%$ efficiency and $1.6 \%$ indels (Fig. 2e, PD- $\Delta 90$ ). The higher efficiency of the PrimeDel strategy may arise from its ability to disrupt the PAM sequences on both strands, which likely increases deletion efficiency by limiting target re-engagement that can lead to indels or re-nicking of the prime-edited strand. Editing efficiencies could be improved for all three approaches by 1.5-fold to 2.5-fold upon the addition of the evoPreQ1 motif the 3' end of the resulting epegRNAs ${ }^{41}$ (Fig. 2e). Together, these data show that twinPE offers a strategy for performing targeted deletions with high flexibility and high product purity that does not rely on the availability of nuclease cut sites.

In a final application of twinPE to mediate targeted deletions, we targeted a therapeutically relevant locus, DMD. Pathogenic DMD alleles, which are responsible for Duchenne muscular dystrophy, commonly contain large deletions in regions containing exons that result in frameshifted mRNA transcripts ${ }^{42}$. Because production of nearly full-length dystrophin protein without replacement of deleted exons can lead to partial rescue of protein function ${ }^{43}$, deletion of a second exon to restore the reading frame has been proposed as a potential therapeutic strategy ${ }^{44}$. We examined three twinPE deletion strategies along with a previously reported Cas9 nuclease deletion strategy for excising exon 51 in $D M D^{45}$. Using single-anchor twinPE deletion approaches, we observed $28 \%$ average efficiency for the deletion of a 780-bp sequence containing exon 51 in DMD (Fig. 2f). While the paired sgRNA Cas9 nuclease strategy for deleting 818 bp achieved higher deletion efficiency (averaging $50 \%$ precise deletion) compared to twinPE strategies, paired Cas9 nuclease-mediated deletion was also accompanied by much higher indel levels of $45 \%$ (33\% desired deletion with additional indels, plus $12 \%$ indels without the desired deletion) compared to twinPE, which averaged $4 \%$ total indels (Fig. 2f).

Exon 51 of $D M D$ could also be excised using alternative spacer pairs that generate a 627-bp deletion with the PrimeDel strategy (30\% average efficiency) or by using twinPE to replace a 589-bp sequence with a 38-bp Bxb1 attB sequence (up to 40\% average efficiency) (Fig. 2f). These experiments show that twinPE and PrimeDel are capable of generating large deletions at therapeutically relevant loci in human cells with far fewer indel byproducts compared to paired Cas9 nuclease strategies.

Both standard prime editing and twinPE require complementarity between the pegRNA spacer and target DNA sequence for Cas9 targeting and nicking, complementarity between 
the pegRNA PBS and the nicked genomic DNA primer for initiation of reverse transcription, and complementarity between the newly synthesized 3' DNA flap and a local DNA sequence (adjacent DNA for standard PE, the second 3' flap for twinPE) for productive prime editing. These three DNA hybridization requirements may explain the observation of reduced offtarget prime editing compared to that of Cas9 nuclease reported by our group and others ${ }^{12,46-}$ 52. To examine potential off-target editing by twinPE, we amplified and sequenced four previously characterized off-target loci for one of the HEK3 spacer sequences following treatment by eight different sets of twinPE reagents targeting $H E K 3$ (SA- $\triangle 77, \mathrm{SA}-\triangle 56, \mathrm{HA}-$ $\Delta 64$, and PD- $\Delta 90$ with standard pegRNAs and epegRNAs). For each of the eight twinPE treatments, no detectable off-target edits or indels were detected at any of the four off-target sites beyond background levels in untreated control samples (Supplementary Table 5).

\section{Targeted DNA integration at safe harbor loci with twinPE and Bxb1 integrase}

Although twinPE can perform larger insertion edits than PE3, the upper limit of sequence insertion with twinPE is insufficient to integrate gene-sized DNA fragments of thousands of base pairs. Having successfully inserted Bxb1 recombinase substrate sequences into endogenous human genomic sites with high efficiency using twinPE, we sought to combine twinPE with serine recombinases for the site-specific integration of DNA cargo (Fig. 3a). Researchers have previously used Bxb1 recombinase to integrate exogenous DNA ranging in size from single genes to entire genetic circuits into genomicallyintegrated attB and attP DNA sequences in a variety of cultured mammalian cells ${ }^{53,54}$ and in Drosophila $^{55}$. To identify sites for DNA cargo integration, we first tested twinPE-mediated insertion of Bxb1 attB and attP attachment sequences at established human genome safe harbor loci in HEK293T cells. We screened 19 spacer pairs targeting the CCR5 locus for insertion of the 38-bp Bxb1 attB sequence (Extended Data Fig. 3). Optimal pegRNAs for six spacer pairs achieved $>50 \%$ editing efficiency of perfectly edited alleles with $3.9-5.4 \%$ indel byproducts (Fig. 3b). Likewise, 32 pegRNA pairs targeting the AAVS1 locus were screened for insertion of the 50-bp Bxb1 attP sequence (Extended Data Fig. 4), of which 17 optimal spacer combinations achieved $>50 \%$ efficiency of perfectly edited alleles with a median of $6.6 \%$ indels (Fig. 3c). Notably, twinPE outperformed PE3 for the replacement of endogenous sequence at CCR5 in HEK293T cells with Bxb1 recombinase attB sites. TwinPE yielded $62 \%$ perfectly edited alleles with 3.3\% indels at one CCR5 site, and 67\% perfectly edited alleles with $4.3 \%$ indels at a second CCR5 site, compared to $3.3 \%$ perfectly edited alleles with $0.1 \%$ 
indels at the first site and 25\% perfectly edited alleles with $1.8 \%$ indels at the second site by PE3 (Extended Data Fig. 5). These results demonstrate that twinPE can be used to insert recombinase substrate sequences at safe harbor loci in human cells with high efficiency.

Next, we examined if twinPE-incorporated attB or attP sequences could serve as target substrates for the BxB1-mediated integration of DNA plasmids containing partner attP or attB sequences. First, we used twinPE to generate single-cell HEK293T clones bearing homozygous attB site insertions at the CCR5 locus. Transfection of this clonal HEK293T cell line with a plasmid expressing Bxb1 recombinase and a 5.6-kB attP-containing donor DNA plasmid yielded an average of $12-17 \%$ integration events per genome of the 5.6-kB plasmid at the target CCR5 site as measured by ddPCR and comparison with an ACTB reference (Extended Data Fig. 6). This efficiency is consistent with previously reported Bxb1-mediated plasmid integration efficiencies in mammalian cells ${ }^{56}$.

Encouraged by these results, we explored whether twinPE-mediated recombinase site insertion and Bxb1 recombinase-mediated DNA donor integration could be achieved by delivering all necessary components in a single transfection step. Encouragingly, our initial efforts to transfect HEK293T cells with plasmids encoding PE2, both pegRNAs, BxB1, and a 5.6-kB donor plasmid resulted in 1.4-6.8\% knock-in efficiency as measured by ddPCR (Fig. $3 d)$. The anticipated junction sequences containing the expected att $L$ and att $R$ recombination products were confirmed by amplicon sequencing, with very high product purities ranging from 91.6-99.8\% average attL or att $R$ junctions without indels (median $=99.0 \%$ ) (Extended Data Fig. 6).

In an effort to improve one-step plasmid integration efficiency with twinPE and Bxb1 recombinase, we tested the twinPE-mediated incorporation of either attB or attP attachment sites, Bxb1 attachment sites containing the canonical GT central dinucleotide core sequence or an alternative GA dinucleotide core sequence ${ }^{56}$, and pegRNAs encoding varying flap overlap lengths. We found that one-step twinPE and Bxb1-mediated plasmid integration performed better when integrating the attB site compared to the attP site, especially when pegRNAs encoded the full-length attB or attP sequence (3.3\% vs. 0.5\% desired integration, Fig. 3e). In addition, we observed that integrations with attB and attP attachment sites containing the canonical GT core sequence generally led to higher knock-in efficiencies compared to those using the alternative GA core sequences (Fig. 3e). Notably, attachment sites containing canonical and alternative core sequences are orthogonal to one another, 
allowing simultaneous knock-in of an attB-GT donor at an attP-GT site in AAVS1 and GA dinucleotide donors at GA attachment sites in CCR5 (Extended Data Fig. 6).

Reducing the extent of flap overlap between pegRNA-encoded recombinase sequences also improved single-transfection plasmid integration efficiency from $3.3 \pm 0.2 \%$ with 38 base pairs of overlap to $5.5 \pm 0.6 \%$ with 20 base pairs of overlap when inserting attB, or from $0.55 \pm 0.1 \%$ with 50 base pairs of overlap to $4.1 \pm 0.8 \%$ with 30 base pairs of overlap when inserting attP (Fig. 3e). Similar twinPE editing efficiencies were observed for inserting attB sequences with long (38-bp) or short (20-bp) flap overlaps. However, products of recombination between attP-containing donor DNA and pegRNA-encoding plasmid were observed when using the 38-bp overlapping pegRNA pair but were not detected when using the 20-bp flap overlap pegRNA pair (Extended Data Fig. 6). Therefore, enhanced integration efficiency with partially overlapping flaps may arise from reducing or eliminating usage of the pegRNA-expressing plasmid DNA as a substrate for Bxb1 recombinase.

We next evaluated plasmid integration at the $A L B$ locus, which is being used clinically for therapeutic transgene expression in hepatocytes ${ }^{57-59}$. As a result of high albumin production by the liver, therapeutic transgene integration at the $A L B$ locus in a relatively small percentage of hepatocytes in principle could achieve therapeutic levels of protein expression for many loss-of-function diseases ${ }^{57}$. In the case of Factor IX transgene expression, increasing circulating protein levels to just $1 \%$ of normal levels has shown a therapeutic effect for the treatment of hemophilia ${ }^{60}$. We devised a twinPE-enabled strategy targeting intron 1 of $A L B$ in which Bxb1 recombinase mediates the integration of circular DNA containing a splice acceptor sequence followed by the cDNA for a protein of interest. Following integration, splicing of the albumin secretion signal encoded in exon 1 to the therapeutic transgene coding sequence would enable its expression and secretion.

We screened pegRNA pairs for the twinPE-mediated insertion of attB within intron 1 of $A L B$ (Fig. 3f) and identified a spacer combination that achieved $43 \%$ correct insertion of the attB sequence. Single-transfection integration of a mock donor plasmid (a promoter-less copy of EGFP and PuroR-T2A-BFP under control of the EF1 $\alpha$ promoter; 5.6-kB total) at this locus was achieved with 1.3\% efficiency in HEK293T cells (Fig. 3g). In Huh7 cells, we observed $34 \%$ correct insertion of the attB sequence (Fig. 3f) and achieved single transfection knock-in efficiency of $2.6 \%$. Interestingly, knock-in at $A L B$ in Huh7 cells was more efficient than knockin at $C C R 5$ (2.6\% vs. 1.1\%), despite CCR5 knock-in being more efficient than $A L B$ knock-in 
in HEK293T cells (Fig. 3g). The transfection of Huh7 cells with plasmids encoding Bxb1, PE2, an attP-containing donor harboring a splice acceptor followed by the cDNA for human factor IX (hFIX) exons 2-8, and pegRNAs programming the insertion of Bxb1 attB at intron 1 of $A L B$ led to detectable levels of hFIX in conditioned media, whereas no hFIX was detected when the pegRNAs targeted CCR5 instead of $A L B$ (Extended Data Fig. 7). Collectively, these results establish a new method for the insertion of gene-sized DNA sequences into targeted genomic loci in previously unmodified human cells without double strand breaks or HDR.

To assess the possibility of unintended Bxb1-mediated donor integration or sequence modifications at endogenous off-target sites in the human genome, we studied five Bxb1 pseudo-sites for further characterization. Pseudo-sites were selected based on the presence of a minimal Bxb1 recognition motif identified by high-resolution recombinase specificity profiling (ACNACNGNNNNNNCNGTNGT) that is common to both attP and attB substrates ${ }^{61}$. Additionally, candidate pseudo-sites required a central GT dinucleotide to match that of the donor DNA plasmid used in our experiments, as corresponding central dinucleotides are necessary for recombination between attB and att $P^{62}$. To search for undesired sequence modifications, we performed targeted amplicon sequencing for each of the five nominated pseudo-sites from seven different samples that had been treated with a 5.6-kb donor DNA plasmid, twinPE reagents targeting CCR5 or AAVS1, and Bxb1 recombinase. For all pseudosites amplified from treated samples, we observed undetectable levels of indels $(<0.1 \%)$ or near-background levels of indels as compared to untreated controls (Extended Data Fig. 7). To capture donor DNA plasmid integration events at the nominated pseudo-sites, we attempted to PCR amplify predicted integration junctions from treated genomic DNA samples. For each sample and pseudo-site target, we observed no PCR product corresponding to offtarget donor integration, while amplicons reporting on the presence of on-target donor integration were readily observed in all cases (Extended Data Fig. 7). These results demonstrate that twinPE+Bxb1 can mediate precise donor integration at the intended target site without generating detected levels of undesired donor integration or sequence alteration at predicted Bxb1 pseudo-sites in the human genome.

\section{TwinPE and recombinase-mediated large inversions}

Large structural variants are found in many human pathogenic alleles, such as the large $600-\mathrm{kb}$ inversion at the $F 8$ locus that causes $~ 50 \%$ of severe hemophilia $A$ cases $^{63}$. Inspired by the high efficiency of recombinase attachments site insertions using twinPE, we 
reasoned that multiplexing the insertion of both attB and attP Bxb1 attachment sites could be used to correct more complex genetic variants by unidirectional deletion or inversion of the intervening DNA sequence. We first tested whether twinPE and Bxb1 can revert an inverted H2B-EGFP coding sequence that is stably integrated into the HEK293T genome via lentivirus transduction (Extended Data Fig. 8). After transfection of the reporter cells with twinPE and Bxb1, we observe up to 19\% GFP positive cells by flow cytometry, indicating successful inversion (Extended Data Fig. 8).

To test the combined twinPE-recombinase inversion approach on a therapeutically relevant locus, we performed a 40-kb inversion between IDS and its pseudogene IDS2. Inversions between these sites have been observed in $\sim 13 \%$ of Hunter syndrome patients ${ }^{64}$, and characterization of the breakpoints in pathogenic alleles revealed that the inversion often occurs within a recombination hotspot that is present in both IDS and IDS $2^{64}$. We targeted regions flanking these recombination hotspots to insert attB and attP sequences, which when oriented in opposing directions can be used as substrates for unidirectional inversion by Bxb1 recombinase to correct the pathogenic allele (Fig. 4a). After screening pegRNA spacer pairs and optimizing pegRNA sequence features, we found pegRNA pairs that are capable of inserting attB or attP attachment sites at the left and right targeted sites with $70 \%$ and $74 \%$ efficiency, respectively in HEK293T cells (Fig. 4b).

Next, we explored multiplexing twinPE-mediated insertion of both attB and attP attachment sites with Bxb1 recombinase-mediated inversion of the 40-kb sequence in IDS and IDS2. Initially, we explored sequential DNA transfections with twinPE components followed by Bxb1 recombinase. A first set of pegRNAs (set 1) was tested that installs a reverse-oriented attP sequence in intron 3 of IDS2 and a forward-oriented attB sequence in intron 7 of IDS. In addition, a second set of pegRNAs (set 2) was used to install a forwardoriented attP sequence in IDS2 and a reverse-oriented attB sequence in IDS. We observed $52 \%$ or $55 \%$ attP sequence insertion and $28 \%$ or $31 \%$ attB sequence insertion for multiplexed editing with pegRNA set 1 and set 2, respectively (Fig. 4c). When edited cells were subsequently transfected with Bxb1 recombinase, we observed a significantly decreased percentage of amplified alleles containing attP and attB sequence compared to mock transfection controls ( $p$-value < 0.05), suggesting that some edited alleles were used as substrates for Bxb1-mediated recombination (Fig. 4c). Amplification of the anticipated inversion junctions followed by amplicon sequencing revealed the presence of both att $L$ and 
attR Bxb1 recombination products with product purity at or above $80 \%$ at both junctions (Fig. $4 d)$.

To carry out one-step twinPE and Bxb1-mediated inversion and circumvent unwanted recombination between pegRNA plasmid DNA, we nucleofected all-RNA components comprising PE2 mRNA, synthetic pegRNAs from set 1, and Bxb1 mRNA. Using amplicon sequencing, we captured the expected inverted allele junctions containing att $R$ and att $L$ sequences (Extended Data Fig. 9). To quantify inversion efficiency, we designed a reverse primer that can bind to an identical sequence in both the non-inverted and inverted alleles and therefore amplify both edits using the same primer pair (Extended Data Fig. 9). We observed 7.7-9.6\% and 2.1-2.6\% inversion efficiency for sequential and one-step methods, respectively (Fig. 4e). Collectively, these data suggest that combining twinPE with sitespecific serine recombinases can be used to correct a common 39-kb inversion found in Hunter syndrome alleles and may eventually serve as a therapeutic strategy for correcting other large or complex pathogenic gene variants.

\section{Discussion}

We developed a twin prime editing approach that can be used to replace, insert, or delete DNA sequences at targeted locations in the genomes of human cells. First, we showed that twinPE can efficiently replace endogenous genomic DNA sequences with exogenous sequences containing $B x b 1$ attB or attP recombination sites with observed editing efficiencies of $>80 \%$ at HEK3 and $>40 \%$ in four different genomic loci in HeLa, U2OS, and K562 cells (Extended Data Fig. 10). An active RT is required to achieve these edits, as Cas9 nickase (H840A) and Cas9 nickase fused to an inactivated RT (K103L/R110S) both failed to generate the desired edits at all sites tested, in contrast to efficient editing with twinPE (Extended Data Fig. 10c). We further used twinPE to recode portions of exon sequences within $P A H$, which in principle could be used to correct multiple pathogenic variants associated with different phenylketonuria patient cohorts using a single prime editor and pegRNA pair. Moreover, since twinPE does not require pegRNAs with RT templates that possess homology to the target site, a feature that is typically required for standard prime editing, and because each RT template does not need to possess the entire replacement sequence, twinPE pegRNAs can encode larger insertions for a given RT template length, including the insertion of a 108-bp sequence with $16 \%$ efficiency using twinPE, representing a 20-fold improvement over PE3. 
We also established precise and flexible twinPE deletion strategies, which make use of the programmability of 3' flap sequences that can be used to fully specify deletion junctions. Previously, this was a limitation of nuclease-based deletions strategies, which are largely limited to deletions between the cut sites and therefore nuclease accessibility. Using twinPE, we tested three deletion strategies, including the previously reported PrimeDel strategy $^{38}$. TwinPE achieved precise deletions of up to 780-nt in DMD sequences including the deletion of exon 51 with up to $28 \%$ efficiency and $4 \%$ total indels. Although Cas 9 nuclease-mediated deletion at this site can achieve $50 \%$ deletion efficiency, Cas 9 nuclease generates $45 \%$ indels in the process. TwinPE thus may be especially useful for applications in which uncontrolled indel byproducts are undesired.

Although efficient targeting of both DNA protospacers is likely necessary for achieving efficient twinPE, we observed only weak correlations between in silico predictions of Cas 9 spacer activity and observed twinPE editing efficiencies (Supplementary Note 3). This poor correlation could arise from other determinants of twinPE efficiency beyond the protospacer, including RT template and PBS choice. While PBS optimization was important for high editing efficiency at many sites, we did not observe an optimal PBS melting temperature across the pegRNA designs in this study. A correlation between prime editing efficiency and the distance between pegRNA-induced nicks was observed, which may suggest an optimal spacing of 50 to $100 \mathrm{bp}$, although many exceptions exist (Supplementary Note 3). Finally, the use of epegRNAs instead of canonical pegRNAs improved twinPE editing efficiency in many cases (Fig. 2c, 2e, and Extended Data Fig. 9c). Therefore, empirical testing of epegRNA pairs will continue to be important to optimize the efficiency of twinPE.

When combined with site-specific serine recombinase enzymes, twinPE can also support the integration of large DNA cargo, or the inversion of DNA sequence. To enable targeted integration of gene-sized DNA plasmids, we combined twinPE and Bxb1 recombinase in a single transfection and successfully inserted a 5.6-kb DNA donor plasmid into the genome of human cells at safe harbor loci $A A V S 1, C C R 5$, and $A L B$ with up to $6.8 \%$, $6.1 \%$, and $2.6 \%$ efficiency, respectively. Notably, Bxb1-mediated knock-in of an attP donor into a HEK293T clonal cell line bearing homozygous attB integration peaked at $17 \%$ in our hands, consistent with observations from others ${ }^{56}$. Given that twinPE-mediated insertions of attB and attP sequences already can exceed $80 \%$, improving the activity of Bxb1 integrase is likely the best opportunity to enhance overall donor knock-in efficiencies. As Bxb1 has been used to insert up to $27 \mathrm{kB}$ in mammalian cells ${ }^{54}$, even larger insertions may be possible. 
Notably, the orthogonality of dinucleotide core sequences may enable gene-sized insertions to be multiplexed to allow simultaneous targeting of two loci with two different donors.

The methods developed here should be applicable for genome engineering and could be applied in the future for the targeted integration of therapeutic genes. TwinPE and Bxb1mediated recombination offer advantages over other approaches. The serine integrase phiC $31^{65}$ and fusions of zinc fingers or dCas 9 to the catalytic domain of Gin recombinase $35,66,67$ have been used to integrate or excise DNA at endogenous pseudo-sites in the human genome, but the extensive sequence preferences inherent to these recombinases greatly limits the number of targetable genomic loci to a minute fraction of genomic loci. The programmable integration of attachment sites by twinPE overcomes many of these challenges by enabling insertion of cognate recombinase recognition sequences at any PE-targetable locus. By comparison to nuclease-based methods, the twinPE and recombinase approach avoids the generation of DSBs, which typically lead to uncontrolled mixtures of by products ${ }^{19}$, and can also lead to chromosomal rearrangements ${ }^{23}$, chromothripsis $^{24}$, large deletions ${ }^{20-22}$, and p53 activation ${ }^{25-27}$. Integration orientation using twinPE and Bxb1 recombinase is strictly controlled by the directionality of the attB and attP sequences $^{62}$, in contrast to uncontrolled integration orientation using homology-independent repair ${ }^{14}$. Methods that leverage HDR also enable control of sequence orientation, and can achieve efficiencies on the order of 5-10\% without drug selection or suppression of NHEJ ${ }^{68-70}$, but HDR is less efficient than NHEJ in most cell types and typically requires DSBs ${ }^{16,17}$.

Methods have also been developed for making targeted gene-sized insertions through paired nicking of the genome and a donor cassette ${ }^{71,72}$. These approaches do not require double strand breaks, but remain reliant on HDR and supportive cell types ${ }^{72}$.

We used twinPE multiplex insertion of attP and attB with Bxb1 to correct a large sequence inversion at IDS and IDS2 associated with Hunter syndrome, resulting in precise inversion of $40 \mathrm{kB}$ in IDS with $9.6 \%$ editing efficiency via sequential transfection and $2.6 \%$ editing efficiency via one-step RNA nucleofection (Fig. 4e). Although zinc finger nuclease, TALEN, and Cas9 nucleases-based approaches have achieved targeted DNA sequence inversions previously ${ }^{73-75}$, DSBs-induced repair pathways also generate undesired products and can lead to de-novo structural variants, including deletion of the targeted DNA sequence. TwinPE and Bxb1 circumvented the issues of nuclease-induced DSBs and enable comparable (19\% by TwinPE+Bxb1 vs. $23 \%$ by Cas $9^{75}$ for a $1.2-k b$ inversion) or higher inversion efficiencies $\left(9.6 \%\right.$ by TwinPE+Bxb1 for $40-k b$ vs. $1.4 \%$ by TALEN ${ }^{74}$ for a $140-k b$ 
inversion) through a precise series of reactions that circumvent the uncontrolled nature of DSB repair.

TwinPE thus expands the capabilities of prime editing to include targeted deletion, replacement, integration, or inversion of larger DNA sequences at specified locations in the genome. By combining twinPE and Bxb1, we achieved gene-size DNA integration and inversion without double-strand breaks, which may provide a strategy to potentially treat genetic diseases arising from loss-of-function or complex structural mutations. Except for spacer sequences and the 8-13 base pairs needed to prime reverse transcription, pegRNAs and donor plasmids in our method do not require homology to the genome. Additional research is needed to fully understand the cellular determinants of twinPE outcomes. Performing large cellular screens to unveil DNA repair components that suppress or enhance twinPE editing efficiency could illuminate mechanistic details that further improve this approach. Future studies on orchestrating twinPE and Bxb1 activity through engineering either or both components could augment the scope and efficiency of twinPE and recombinase editing in mammalian cells. 


\section{References}

1 Auton, A. et al. A global reference for human genetic variation. Nature 526, 68-74, doi:10.1038/nature15393 (2015).

2 Landrum, M. J. et al. ClinVar: public archive of relationships among sequence variation and human phenotype. Nucleic Acids Res 42, D980-985, doi:10.1093/nar/gkt1113 (2014).

3 Weischenfeldt, J., Symmons, O., Spitz, F. \& Korbel, J. O. Phenotypic impact of genomic structural variation: insights from and for human disease. Nat Rev Genet 14, 125-138, doi:10.1038/nrg3373 (2013).

4 Cox, D. B., Platt, R. J. \& Zhang, F. Therapeutic genome editing: prospects and challenges. Nat Med 21, 121-131, doi:10.1038/nm.3793 (2015).

5 Doudna, J. A. The promise and challenge of therapeutic genome editing. Nature $\mathbf{5 7 8}$, 229-236, doi:10.1038/s41586-020-1978-5 (2020).

6 Anzalone, A. V., Koblan, L. W. \& Liu, D. R. Genome editing with CRISPR-Cas nucleases, base editors, transposases and prime editors. Nat Biotechno/ 38, 824-844, doi:10.1038/s41587-020-0561-9 (2020).

7 Jinek, M. et al. A Programmable Dual-RNA-Guided DNA Endonuclease in Adaptive Bacterial Immunity. Science 337, 816-821, doi:10.1126/science.1225829 (2012).

8 Cong, L. et al. Multiplex genome engineering using CRISPR/Cas systems. Science (New York, N.Y.) 339, 819-823, doi:10.1126/science.1231143 (2013).

9 Mali, P. et al. RNA-guided human genome engineering via Cas9. Science 339, 823826, doi:10.1126/science.1232033 (2013).

10 Komor, A. C., Kim, Y. B., Packer, M. S., Zuris, J. A. \& Liu, D. R. Programmable editing of a target base in genomic DNA without double-stranded DNA cleavage. Nature 533, 420-424, doi:10.1038/nature17946 (2016).

11 Gaudelli, N. M. et al. Programmable base editing of $A^{*} T$ to $G^{*} C$ in genomic DNA without DNA cleavage. Nature 551, 464-471, doi:10.1038/nature24644 (2017).

12 Anzalone, A. V. et al. Search-and-replace genome editing without double-strand breaks or donor DNA. Nature 576, 149-157, doi:10.1038/s41586-019-1711-4 (2019).

13 Canver, M. C. et al. Characterization of genomic deletion efficiency mediated by clustered regularly interspaced short palindromic repeats (CRISPR)/Cas9 nuclease system in mammalian cells. The Journal of biological chemistry 289, 21312-21324, doi:10.1074/jbc.M114.564625 (2014).

14 Suzuki, K. et al. In vivo genome editing via CRISPR/Cas9 mediated homologyindependent targeted integration. Nature 540, 144-149, doi:10.1038/nature20565 (2016).

15 Wang, B. et al. Highly efficient CRISPR/HDR-mediated knock-in for mouse embryonic stem cells and zygotes. Biotechniques 59, 201-202, 204, 206-208, doi:10.2144/000114339 (2015).

16 Branzei, D. \& Foiani, M. Regulation of DNA repair throughout the cell cycle. Nature Reviews Molecular Cell Biology 9, 297-308, doi:10.1038/nrm2351 (2008).

17 Heyer, W. D., Ehmsen, K. T. \& Liu, J. Regulation of homologous recombination in eukaryotes. Annual review of genetics 44, 113-139, doi:10.1146/annurev-genet051710-150955 (2010).

18 Gasperini, M. et al. CRISPR/Cas9-mediated scanning for regulatory elements required for HPRT1 expression via thousands of large, programmed genomic deletions. The American Journal of Human Genetics 101, 192-205 (2017). 
19 Pawelczak, K. S., Gavande, N. S., VanderVere-Carozza, P. S. \& Turchi, J. J. Modulating DNA Repair Pathways to Improve Precision Genome Engineering. ACS Chem Biol 13, 389-396, doi:10.1021/acschembio.7b00777 (2018).

20 Kosicki, M., Tomberg, K. \& Bradley, A. Repair of double-strand breaks induced by CRISPR-Cas9 leads to large deletions and complex rearrangements. Nature Biotechnology 36, 765-771, doi:10.1038/nbt.4192 (2018).

21 Alanis-Lobato, G. et al. Frequent loss-of-heterozygosity in CRISPR-Cas9-edited early human embryos. Proceedings of the National Academy of Sciences, 202004832, doi:10.1073/pnas.2004832117 (2021).

22 Song, Y. et al. Large-Fragment Deletions Induced by Cas9 Cleavage while Not in the BEs System. Molecular Therapy - Nucleic Acids 21, 523-526, doi:10.1016/j.omtn.2020.06.019 (2020).

23 Brunet, E. \& Jasin, M. Induction of Chromosomal Translocations with CRISPR-Cas9 and Other Nucleases: Understanding the Repair Mechanisms That Give Rise to Translocations. Advances in experimental medicine and biology 1044, 15-25, doi:10.1007/978-981-13-0593-1_2 (2018).

24 Leibowitz, M. L. et al. Chromothripsis as an on-target consequence of CRISPR-Cas9 genome editing. Nature Genetics, doi:10.1038/s41588-021-00838-7 (2021).

25 Haapaniemi, E., Botla, S., Persson, J., Schmierer, B. \& Taipale, J. CRISPR-Cas9 genome editing induces a p53-mediated DNA damage response. Nature Medicine 24, 927-930, doi:10.1038/s41591-018-0049-z (2018).

26 Ihry, R. J. et al. p53 inhibits CRISPR-Cas9 engineering in human pluripotent stem cells. Nature Medicine 24, 939-946, doi:10.1038/s41591-018-0050-6 (2018).

27 Enache, O. M. et al. Cas9 activates the p53 pathway and selects for p53-inactivating mutations. Nat Genet 52, 662-668, doi:10.1038/s41588-020-0623-4 (2020).

28 Kurt, I. C. et al. CRISPR C-to-G base editors for inducing targeted DNA transversions in human cells. Nat Biotechnol 39, 41-46, doi:10.1038/s41587-020-0609-x (2021).

29 Zhao, D. et al. Glycosylase base editors enable C-to-A and C-to-G base changes. Nat Biotechnol 39, 35-40, doi:10.1038/s41587-020-0592-2 (2021).

30 Koblan, L. W. et al. Efficient C-G-to-G•C base editors developed using CRISPRi screens, target-library analysis, and machine learning. Nature Biotechnology, doi:10.1038/s41587-021-00938-z (2021).

31 Grindley, N. D., Whiteson, K. L. \& Rice, P. A. Mechanisms of site-specific recombination. Annu Rev Biochem 75, 567-605, doi:10.1146/annurev.biochem.73.011303.073908 (2006).

32 Merrick, C. A., Zhao, J. \& Rosser, S. J. Serine Integrases: Advancing Synthetic Biology. ACS Synthetic Biology 7, 299-310, doi:10.1021/acssynbio.7b00308 (2018).

33 Sarkar, I., Hauber, I., Hauber, J. \& Buchholz, F. HIV-1 proviral DNA excision using an evolved recombinase. Science 316, 1912-1915, doi:10.1126/science.1141453 (2007).

34 Karpinski, J. et al. Directed evolution of a recombinase that excises the provirus of most HIV-1 primary isolates with high specificity. Nat Biotechnol 34, 401-409, doi:10.1038/nbt.3467 (2016).

35 Chaikind, B., Bessen, J. L., Thompson, D. B., Hu, J. H. \& Liu, D. R. A programmable Cas9-serine recombinase fusion protein that operates on DNA sequences in mammalian cells. Nucleic Acids Res 44, 9758-9770, doi:10.1093/nar/gkw707 (2016).

36 Mercer, A. C., Gaj, T., Fuller, R. P. \& Barbas, C. F., 3rd. Chimeric TALE recombinases with programmable DNA sequence specificity. Nucleic Acids Res 40, 11163-11172, doi:10.1093/nar/gks875 (2012). 
37 Kim, A. I. et al. Mycobacteriophage Bxb1 integrates into the Mycobacterium smegmatis groEL1 gene. Molecular Microbiology 50, 463-473, doi:https://doi.org/10.1046/i.1365-2958.2003.03723.x (2003).

38 Choi, J. et al. Precise genomic deletions using paired prime editing. bioRxiv, 2020.2012.2030.424891, doi:10.1101/2020.12.30.424891 (2021).

39 Lin, Q. et al. High-efficiency prime editing with optimized, paired pegRNAs in plants. Nature Biotechnology, doi:10.1038/s41587-021-00868-w (2021).

40 Scriver, C. R. The PAH gene, phenylketonuria, and a paradigm shift. Human Mutation 28, 831-845, doi:https://doi.org/10.1002/humu.20526 (2007).

41 Nelson, J. W. et al. Engineered pegRNAs improve prime editing efficiency. Nat Biotechnol, doi:10.1038/s41587-021-01039-7 (2021).

42 Flanigan, K. M. et al. Mutational spectrum of DMD mutations in dystrophinopathy patients: application of modern diagnostic techniques to a large cohort. Human mutation 30, 1657-1666, doi:10.1002/humu.21114 (2009).

43 Hoffman, E. P. et al. Restoring dystrophin expression in duchenne muscular dystrophy muscle progress in exon skipping and stop codon read through. Am J Pathol 179, 1222, doi:10.1016/j.ajpath.2011.03.050 (2011).

44 Aartsma-Rus, A. et al. Development of Exon Skipping Therapies for Duchenne Muscular Dystrophy: A Critical Review and a Perspective on the Outstanding Issues. Nucleic acid therapeutics 27, 251-259, doi:10.1089/nat.2017.0682 (2017).

45 Ousterout, D. G. et al. Multiplex CRISPR/Cas9-based genome editing for correction of dystrophin mutations that cause Duchenne muscular dystrophy. Nature Communications 6, 6244, doi:10.1038/ncomms7244 (2015).

46 Kim, D. Y., Moon, S. B., Ko, J.-H., Kim, Y.-S. \& Kim, D. Unbiased investigation of specificities of prime editing systems in human cells. Nucleic Acids Research 48, 10576-10589, doi:10.1093/nar/gkaa764 (2020).

47 Jin, S. et al. Genome-wide specificity of prime editors in plants. Nature Biotechnology, doi:10.1038/s41587-021-00891-x (2021).

48 Habib, O., Habib, G., Hwang, G.-H. \& Bae, S. Comprehensive analysis of prime editing outcomes in human embryonic stem cells. bioRxiv, 2021.2004.2012.439533, doi:10.1101/2021.04.12.439533 (2021).

49 Gao, R. et al. No observable guide-RNA-independent off-target mutation induced by prime editor. bioRxiv, 2021.2004.2009.439109, doi:10.1101/2021.04.09.439109 (2021).

50 Schene, I. F. et al. Prime editing for functional repair in patient-derived disease models. Nature Communications 11, 5352, doi:10.1038/s41467-020-19136-7 (2020).

51 Gao, P. et al. Prime editing in mice reveals the essentiality of a single base in driving tissue-specific gene expression. Genome Biology 22, 83, doi:10.1186/s13059-02102304-3 (2021).

52 Park, S.-J. et al. Targeted mutagenesis in mouse cells and embryos using an enhanced prime editor. Genome Biology 22, 170, doi:10.1186/s13059-021-02389-w (2021).

$53 \mathrm{Xu}, \mathrm{Z}$. et al. Accuracy and efficiency define Bxb1 integrase as the best of fifteen candidate serine recombinases for the integration of DNA into the human genome. BMC Biotechnology 13, 87, doi:10.1186/1472-6750-13-87 (2013).

54 Duportet, X. et al. A platform for rapid prototyping of synthetic gene networks in mammalian cells. Nucleic acids research 42, 13440-13451, doi:10.1093/nar/gku1082 (2014). 
55 Voutev, R. \& Mann, R. S. Bxb1 phage recombinase assists genome engineering in Drosophila melanogaster. Biotechniques 62, 37-38, doi:10.2144/000114494 (2017).

56 Jusiak, B. et al. Comparison of Integrases Identifies Bxb1-GA Mutant as the Most Efficient Site-Specific Integrase System in Mammalian Cells. ACS Synthetic Biology 8, 16-24, doi:10.1021/acssynbio.8b00089 (2019).

57 Sharma, R. et al. In vivo genome editing of the albumin locus as a platform for protein replacement therapy. Blood 126, 1777-1784, doi:10.1182/blood-2014-12-615492 (2015).

58 Barzel, A. et al. Promoterless gene targeting without nucleases ameliorates haemophilia B in mice. Nature 517, 360-364, doi:10.1038/nature13864 (2015).

59 Ascending Dose Study of Genome Editing by Zinc Finger Nuclease Therapeutic SBFIX in Subjects With Severe Hemophilia B.

https://ClinicalTrials.gov/show/NCT02695160.

60 Nathwani, A. C. et al. Long-Term Safety and Efficacy of Factor IX Gene Therapy in Hemophilia B. New Engl J Med 371, 1994-2004, doi:10.1056/NEJMoa1407309 (2014).

61 Bessen, J. L. et al. High-resolution specificity profiling and off-target prediction for sitespecific DNA recombinases. Nature Communications 10, 1937, doi:10.1038/s41467019-09987-0 (2019).

62 Ghosh, P., Kim, A. I. \& Hatfull, G. F. The Orientation of Mycobacteriophage Bxb1 Integration Is Solely Dependent on the Central Dinucleotide of attP and attB. Molecular Cell 12, 1101-1111, doi:10.1016/S1097-2765(03)00444-1 (2003).

63 Graw, J. et al. Haemophilia A: from mutation analysis to new therapies. Nat Rev Genet 6, 488-501, doi:10.1038/nrg1617 (2005).

64 Bondeson, M. L. et al. Inversion of the IDS gene resulting from recombination with IDS-related sequences is a common cause of the Hunter syndrome. Human molecular genetics 4, 615-621, doi:10.1093/hmg/4.4.615 (1995).

65 Guha, T. K. \& Calos, M. P. Nucleofection of phiC31 Integrase Protein Mediates Sequence-Specific Genomic Integration in Human Cells. J Mol Biol 432, 3950-3955, doi:10.1016/j.jmb.2020.04.019 (2020).

66 Gaj, T., Mercer, A. C., Sirk, S. J., Smith, H. L. \& Barbas, C. F., 3rd. A comprehensive approach to zinc-finger recombinase customization enables genomic targeting in human cells. Nucleic acids research 41, 3937-3946, doi:10.1093/nar/gkt071 (2013).

67 Gaj, T. et al. Enhancing the Specificity of Recombinase-Mediated Genome Engineering through Dimer Interface Redesign. J Am Chem Soc 136, 5047-5056, doi:10.1021/ja4130059 (2014).

68 Ruan, J. et al. Highly efficient CRISPR/Cas9-mediated transgene knockin at the H11 locus in pigs. Scientific Reports 5, 14253, doi:10.1038/srep14253 (2015).

69 Chu, V. T. et al. Increasing the efficiency of homology-directed repair for CRISPRCas9-induced precise gene editing in mammalian cells. Nature Biotechnology 33, 543548, doi:10.1038/nbt.3198 (2015).

70 Zhang, J.-P. et al. Efficient precise knockin with a double cut HDR donor after CRISPR/Cas9-mediated double-stranded DNA cleavage. Genome Biology 18, 35, doi:10.1186/s13059-017-1164-8 (2017).

71 Chen, $X$. et al. In trans paired nicking triggers seamless genome editing without double-stranded DNA cutting. Nat Commun 8, 657, doi:10.1038/s41467-017-00687-1 (2017).

72 Hyodo, T. et al. Tandem Paired Nicking Promotes Precise Genome Editing with Scarce Interference by p53. Cell Reports 30, 1195-1207.e1197, doi:https://doi.org/10.1016/i.celrep.2019.12.064 (2020). 
73 Lee, H. J., Kweon, J., Kim, E., Kim, S. \& Kim, J. S. Targeted chromosomal duplications and inversions in the human genome using zinc finger nucleases. Genome Res 22, 539-548, doi:10.1101/gr.129635.111 (2012).

74 Park, C. Y. et al. Targeted inversion and reversion of the blood coagulation factor 8 gene in human iPS cells using TALENs. Proc Natl Acad Sci U S A 111, 9253-9258, doi:10.1073/pnas.1323941111 (2014).

$75 \mathrm{Li}, \mathrm{J}$. et al. Efficient inversions and duplications of mammalian regulatory DNA elements and gene clusters by CRISPR/Cas9. Journal of molecular cell biology 7 , 284-298, doi:10.1093/jmcb/mjv016 (2015).

76 Clement, K. et al. CRISPResso2 provides accurate and rapid genome editing sequence analysis. Nature Biotechnology 37, 224-226, doi:10.1038/s41587-019-00323 (2019).

77 Clement, K., Farouni, R., Bauer, D. E. \& Pinello, L. AmpUMI: design and analysis of unique molecular identifiers for deep amplicon sequencing. Bioinformatics 34, i202i210, doi:10.1093/bioinformatics/bty264 (2018).

78 Shen, W., Le, S., Li, Y. \& Hu, F. SeqKit: A Cross-Platform and Ultrafast Toolkit for FASTA/Q File Manipulation. PLoS One 11, e0163962, doi:10.1371/journal.pone.0163962 (2016).

79 Levy, J. M. \& Nicoll, R. A. Membrane-associated guanylate kinase dynamics reveal regional and developmental specificity of synapse stability. The Journal of Physiology 595, 1699-1709, doi:https://doi.org/10.1113/JP273147 (2017).

80 Koblan, L. W. et al. In vivo base editing rescues Hutchinson-Gilford progeria syndrome in mice. Nature 589, 608-614, doi:10.1038/s41586-020-03086-7 (2021).

81 Gaudelli, N. M. et al. Directed evolution of adenine base editors with increased activity and therapeutic application. Nature Biotechnology 38, 892-900, doi:10.1038/s41587020-0491-6 (2020). 


\section{Acknowledgements}

We thank Erik Sontheimer's group for sharing Huh7 cells and Liu Lab members for helpful discussions. This work was supported by the Merkin Institute of Transformative Technologies in Healthcare, US NIH grants U01 Al142756, RM1 HG009490, and R35 GM118062, and the HHMI. A.V.A. acknowledges a Jane Coffin Childs postdoctoral fellowship through the HHMI.

\section{Author contributions}

A.V.A., X.D.G., C.J.P., A.T.N., and J.M.L. designed experiments. A.V.A., X.D.G., C.J.P., A.T.N., L.W.K., A.R., and J.A.M.M. performed experiments and analyzed data. A.V.A., X.D.G., C.J.P. and D.R.L. wrote the manuscript. D.R.L. supervised the research.

\section{Declaration of Interests}

D.R.L. is a consultant and equity holder of Beam Therapeutics, Prime Medicine, Pairwise Plants, and Chroma Medicine, companies that use genome editing or genome engineering technologies. A.V.A., C.J.P., and J.M.L. are currently employees at Prime Medicine. The authors have filed patent applications on twinPE and prime editing through the Broad Institute. 


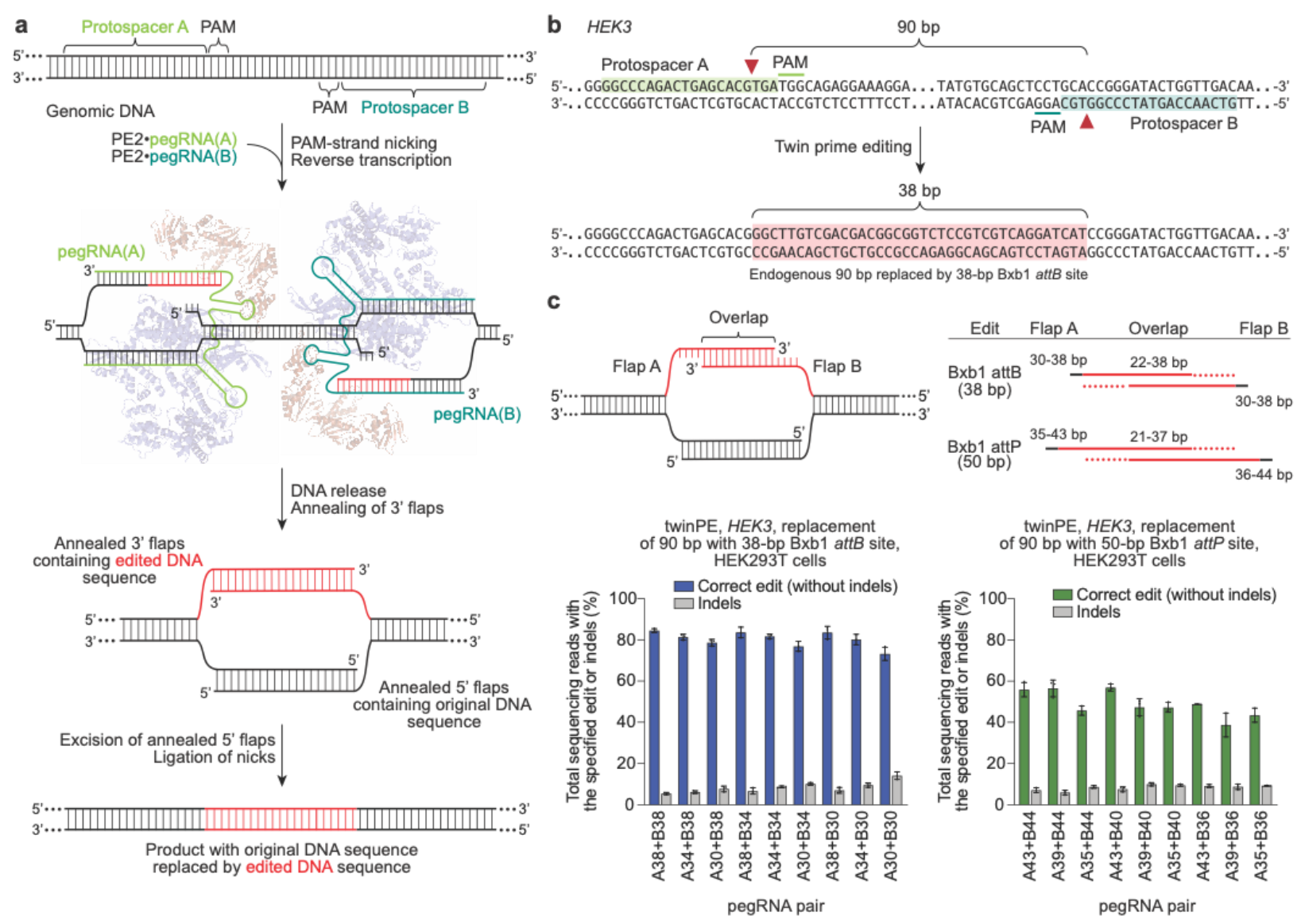

Figure 1 | Overview of twinPE and twinPE-mediated sequence replacement. (a) TwinPE systems target genomic DNA sequences that contain two protospacer sequences on opposite strands of DNA. PE2•pegRNA complexes target each protospacer, generate a single-stranded nick, and reverse transcribe the pegRNA-encoded template containing the desired insertion sequence. After synthesis and release of the 3' DNA flaps, a hypothetical intermediate exists possessing annealed 3' flaps containing the edited DNA sequence and annealed 5' flaps containing the original DNA sequence. Excision of the original DNA sequence contained in the 5' flaps, followed by ligation of the 3' flaps to the corresponding excision sites, generates the desired edited product. (b) Example of twinPE-mediated replacement of a 90-bp sequence in $H E K 3$ with a 38-bp Bxb1 attB sequence. (c) Evaluation of twinPE in HEK293T cells for the installation of the 38-bp Bxb1 attB site as shown in (b) or the 50-bp Bxb1 attP site at $H E K 3$ using pegRNAs that template varying lengths of the insertion sequence. pegRNA names indicate spacer (A or B) and length of RT template. Values and error bars reflect the mean and s.d. of three independent biological replicates. 
bioRxiv preprint doi: https://doi.org/10.1101/2021.11.01.466790; this version posted November 2, 2021. The copyright holder for this preprint (which was not certified by peer review) is the author/funder, who has granted bioRxiv a license to display the preprint in perpetuity. It is made available under aCC-BY-NC 4.0 International license.

a Twin prime editing
(this work)

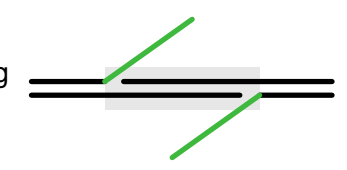

PrimeDel (Choi et al.)

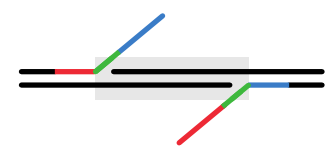

Paired pegRNAs (Lin et al.)

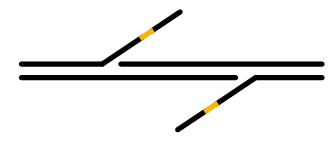

C $=$ excised region $\longrightarrow$ and $\longrightarrow=\begin{gathered}\text { homologous } \\ \text { sequence }\end{gathered}$

PAH exon 4, 64 bp recoded, twinPE, HEK293T cells

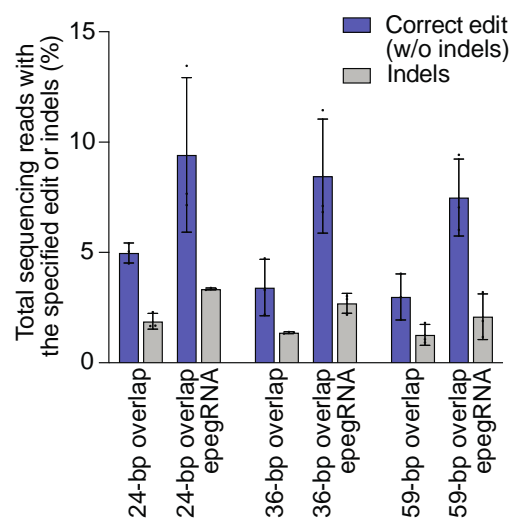

d

Single-anchor twinPE (SA)

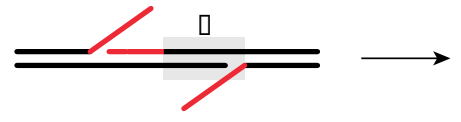

Hybrid-anchor twinPE (HA)

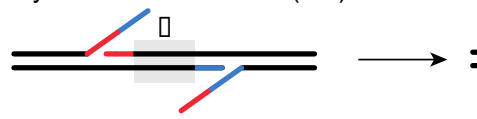

PrimeDel (PD)

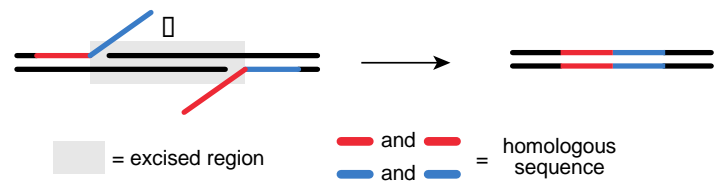

f

$\begin{array}{lllll}\text { Deletion size (bp) } & 780 & 780 & 780 & 818\end{array}$

Editing strategy SA-1 SA-2 SA-3 Cas9

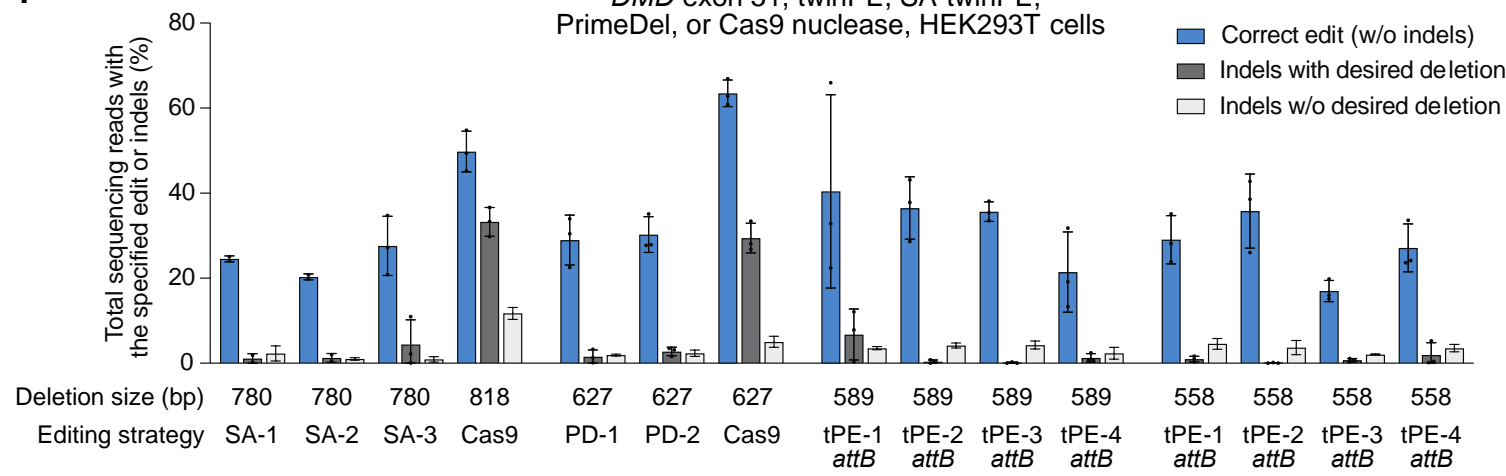

$D M D$ exon 51, twinPE, SA-twinPE,

PrimeDel, or Cas9 nuclease, HEK293T cells

HEK3, SA-twinPE, HA-twinPE, or PrimeDel, HEK293T cells

, 7,64 bp recoded, twinPE, HEK293T cells

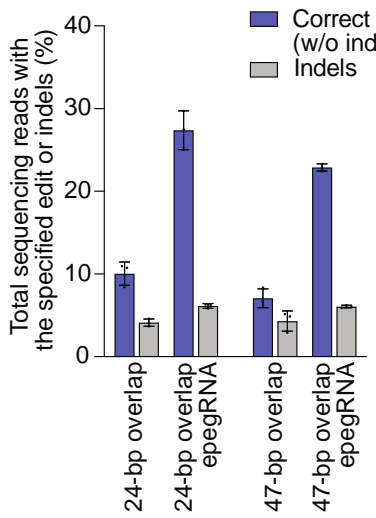

e

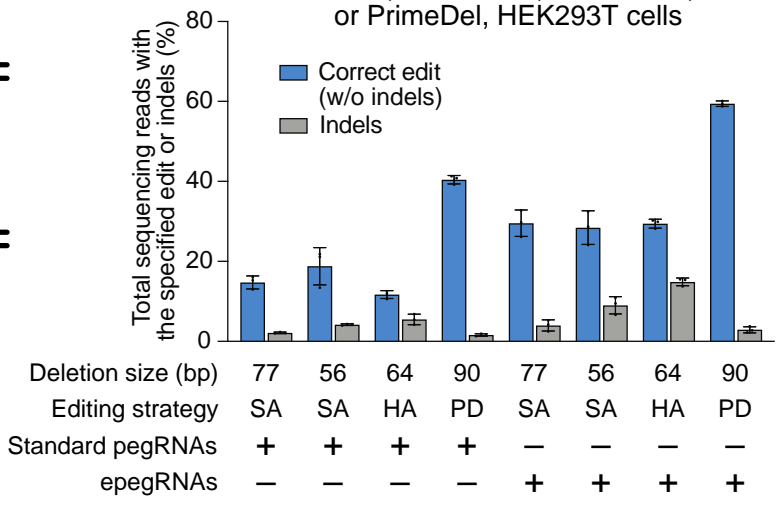


Figure 2 | Targeted sequence insertion, deletion, and recoding with twinPE in human cells. (a) Schematic diagram illustrating designs and edits generated for twinPE, PrimeDel ${ }^{36}$ and paired pegRNAs ${ }^{37}$. Shaded gray boxes indicate regions where DNA is excised, green lines indicate the incorporation of heterologous DNA sequence, red and blue lines indicate regions of sequence homology (red to red, blue to blue), and yellow lines indicate regions with small edits. PrimeDel can introduce but does not require introduction of heterologous sequence (green). (b) Insertion of FKBP coding sequence fragments with PE3 (12 bp, $36 \mathrm{bp}$, $108 \mathrm{bp}$ ) or twinPE (108 bp) at HEK3 in HEK293T cells. (c) Recoding of sequence within exons 4 and 7 in $P A H$ in HEK293T cells using twinPE. A 64-bp target sequence in exon 4 was edited using 24, 36, or 59 bp of overlapping flaps, a 46-bp target sequence in exon 7 was edited using 22 or 42 bp of overlapping flaps, or a 64-bp sequence in exon 7 was edited using 24 or $47 \mathrm{bp}$ of overlapping flaps. Editing activity was compared using standard pegRNAs or epegRNAs containing 3' evoPreQ1 motifs. (d) Schematic diagram showing three distinct dual-flap deletion strategies that were investigated for carrying out targeted deletions. The "Single-anchor (SA)" twinPE strategy allows for flexible deletion starting at an arbitrary position 3' of one nick site and ending at the other nick site. The "Hybrid-anchor (HA)" twinPE strategy allows for flexible deletion of sequence at arbitrarily chosen positions between the two nick sites. The "PrimeDel (PD)" strategy of Shendure and co-workers tested here allows for deletion of the sequence starting at one nick site and ending at another nick site. Shaded gray boxes indicate regions where DNA is excised, red and blue lines indicate regions of sequence homology (red to red, blue to blue). (e) Deletion of sequences at $H E K 3$ in HEK293T cells using the SA-twinPE, HA-twinPE, or PD strategies targeting the same protospacer pair. Editing activity was compared using standard pegRNAs or epegRNAs containing 3' evoPreQ1 motifs. (f) Deletion of exon 51 sequence at the DMD locus in HEK293T cells using SA-twinPE, PD, paired Cas9 nuclease, or twinPE-mediated attB sequence replacement. A unique molecular identifier (UMI) protocol was applied to remove PCR bias (see Supplementary Note 1). Values and error bars in (b-f) reflect the mean and s.d. of three independent biological replicates. 
a

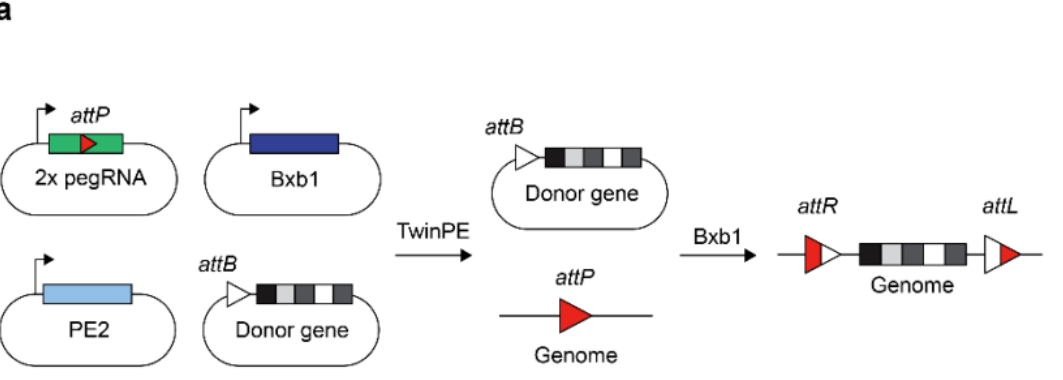

C

AAVS1, Bxb1 attP insertion,

HEK293T cells

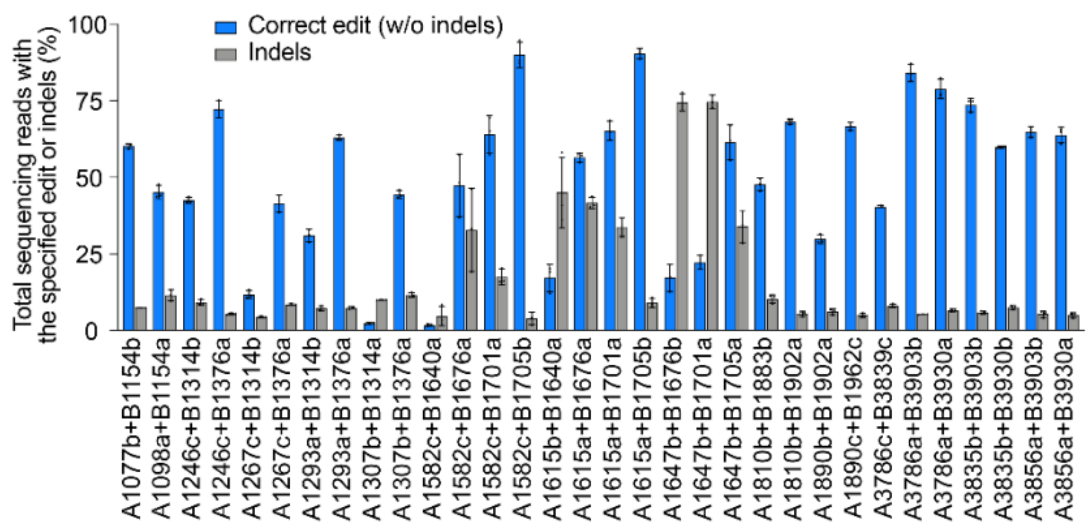

b CCR5, Bxb1 attB insertion,

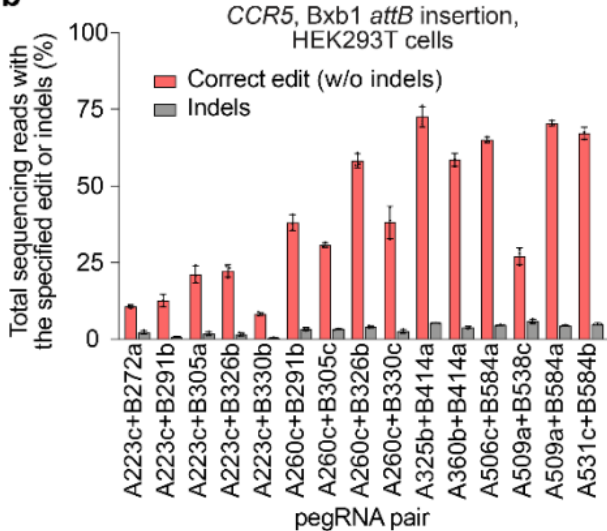

pegRNA pair

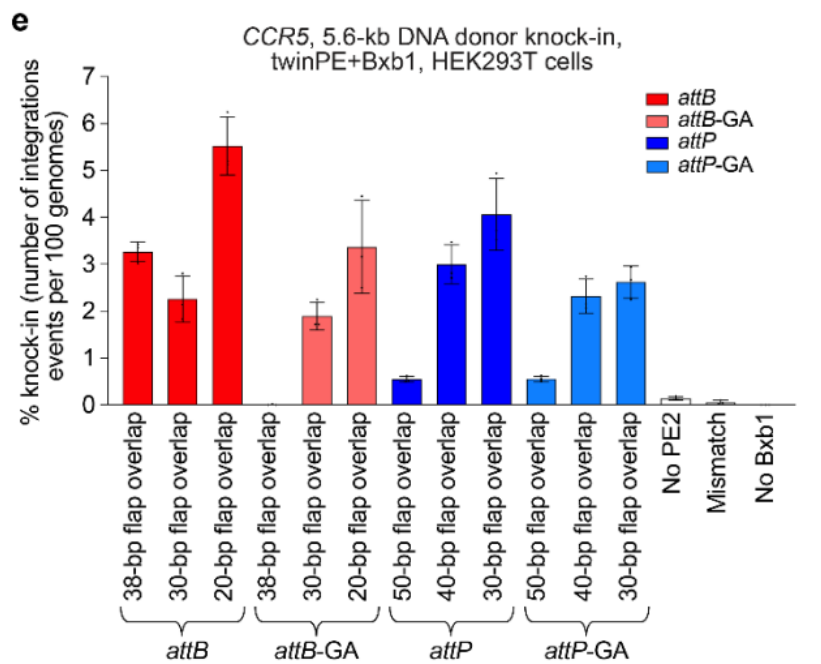

f

d
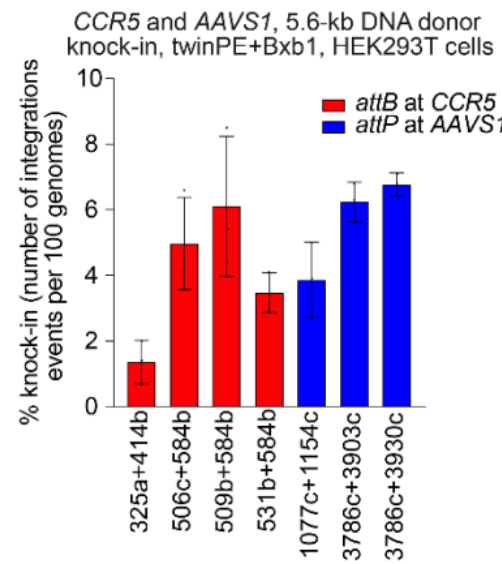

ALB, Bxb1 attB insertion,
HEK293T cells or Huh7 cells pegRNA pair

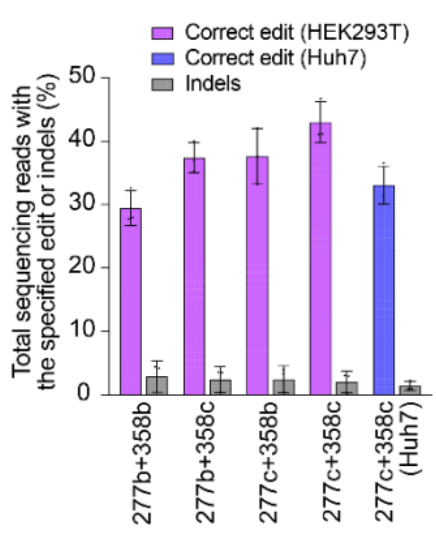

g CCR5 or ALB, 5.6-kb DNA donor knock-in, twinPE+Bxb1, HEK293T cells or Huh7 cells

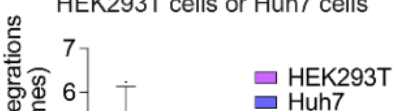

Figure 3 | Site-specific genomic integration of DNA cargo with twinPE and Bxb1 recombinase in human cells. (a) Schematic diagram of twinPE and Bxb1 recombinasemediated site-specific genomic integration of DNA cargo. (b) Screening of twinPE pegRNA pairs for insertion of the Bxb1 attB sequence at the CCR5 locus in HEK293T cells. (c) Screening of twinPE pegRNA pairs for installation of the Bxb1 attP sequence at the AAVS1 locus in HEK293T cells. (d) Single transfection knock-in of 5.6-kb DNA donors using twinPE pegRNA pairs targeting CCR5 (red) or AAVS1 (blue). The twinPE pegRNAs install attB at $C C R 5$ or attP at $A A V S 1$. Bxb1 integrates a donor bearing the corresponding attachment site into the genomic attachment site. The number of integration events per 100 genomes is defined as the ratio of the target amplicon spanning the donor-genome junction to a reference 
amplicon in $A C T B$, as determined by ddPCR. (e) Optimization of single-transfection integration at CCR5 using the A531+B584 spacers for the twinPE pegRNA pair. Identity of the templated edit (attB or attP), identity of the central dinucleotide (wild-type GT or orthogonal mutant GA), and length of the overlap between flaps were varied to identify combinations that supported the highest integration efficiency. \% knock-in quantified as in (d). (f) Pairs of pegRNAs were assessed for their ability to insert Bxb1 attBinto the first intron of ALB. Protospacer sequences (277 and 358 ) are constant across the pegRNA pairs. The pegRNAs vary in their PBS lengths (variant b or c). The $277 \mathrm{c} / 358 \mathrm{c}$ pair that performs best in HEK293T cells can also introduce the desired edit in Huh7 cells. (g) Comparison of single transfection knock-in efficiencies at CCR5 and ALB in HEK293T and Huh7 cell lines. \% knock-in quantified as in (d). Values and error bars reflect the mean and s.d. of three independent biological replicates. 
a

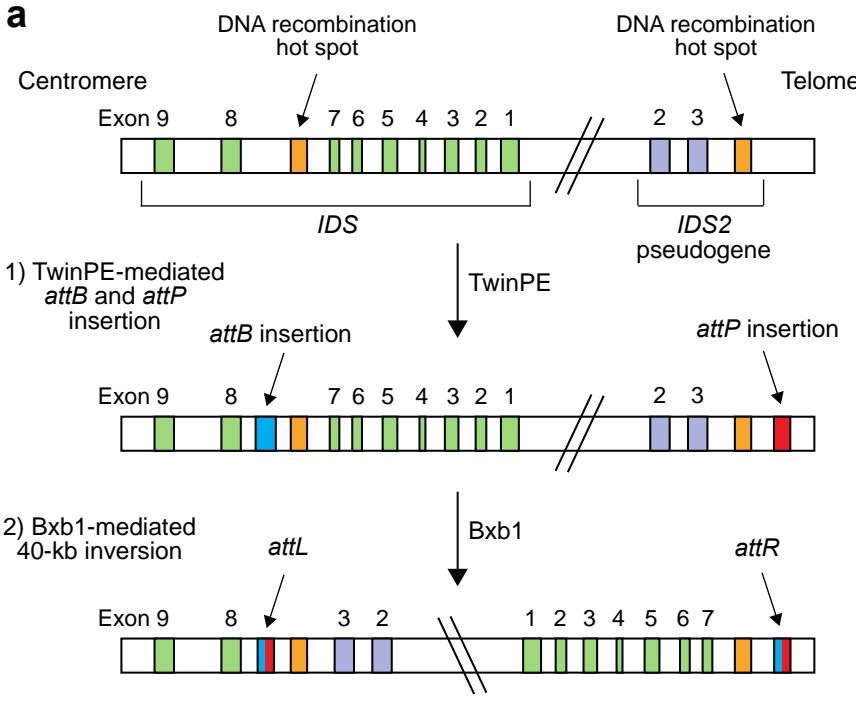

b

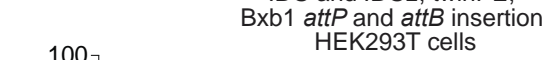

$\begin{array}{lllllllllllll}\text { Spacer } 1 & \text { A1 } & \text { A1 } & \text { A1 } & \text { A1 } & \text { A1 } & \text { A1 } & \text { A4 } & \text { A4 } & \text { C2 } & \text { C2 } & \text { C2 } & \text { C2 }\end{array}$ $\begin{array}{lllllllllllll}\text { Spacer } 2 & \text { B2 } & \text { B2 } & \text { B3 } & \text { B3 } & \text { B4 } & \text { B4 } & \text { B7 } & \text { B7 } & \text { D1 } & \text { D1 } & \text { D2 } & \text { D2 }\end{array}$ attP-fwd -+-+-+-+attP-rev +-+-+-+--attB-fwd - $-\quad-\quad-\quad-\quad-\quad+\quad+-$

C $\begin{array}{cl}\text { IDS and IDS2, twinPE } & \square \text { Correct attP (w/o indels) } \\ \begin{array}{c}\text { I sequential transfection of Bxb1, } \\ \text { HEK293T cells }\end{array} & \square \text { Correct attB (w/o indels) } \\ & \square \text { Indels }\end{array}$

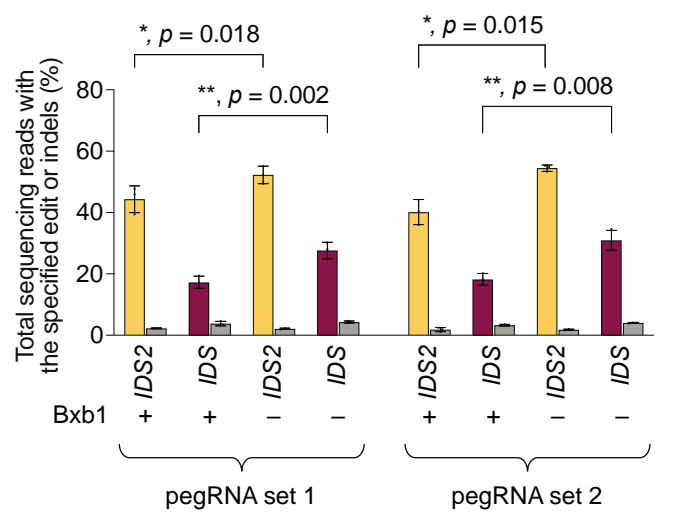

e
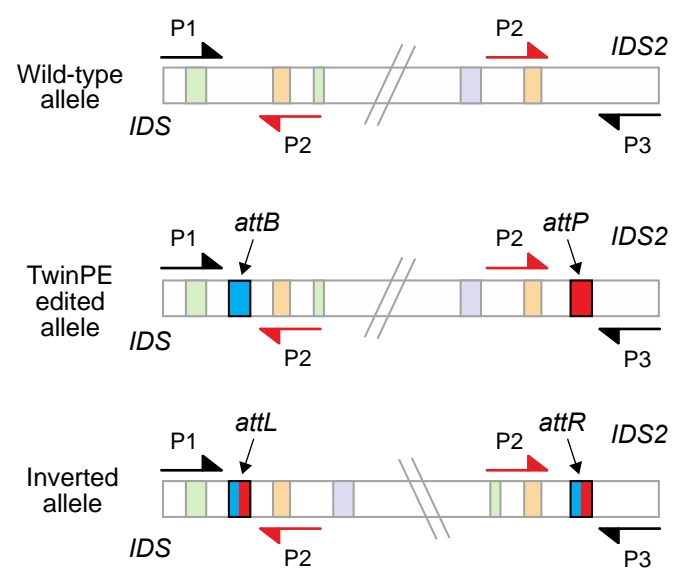

d PCR of predicted
inversion junctions<smiles>[R]C1C2C=CC(C2)CS1(=O)=O</smiles><smiles>C1CCCCC1</smiles>

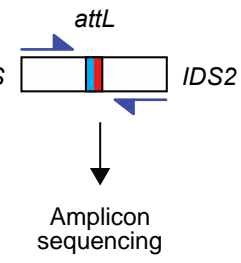

IDS and IDS2, twinPE+Bxb1 40-kb inversion, sequential transfection, HEK293T' cells

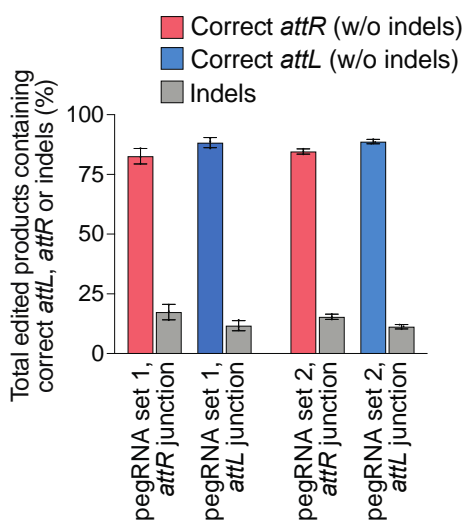

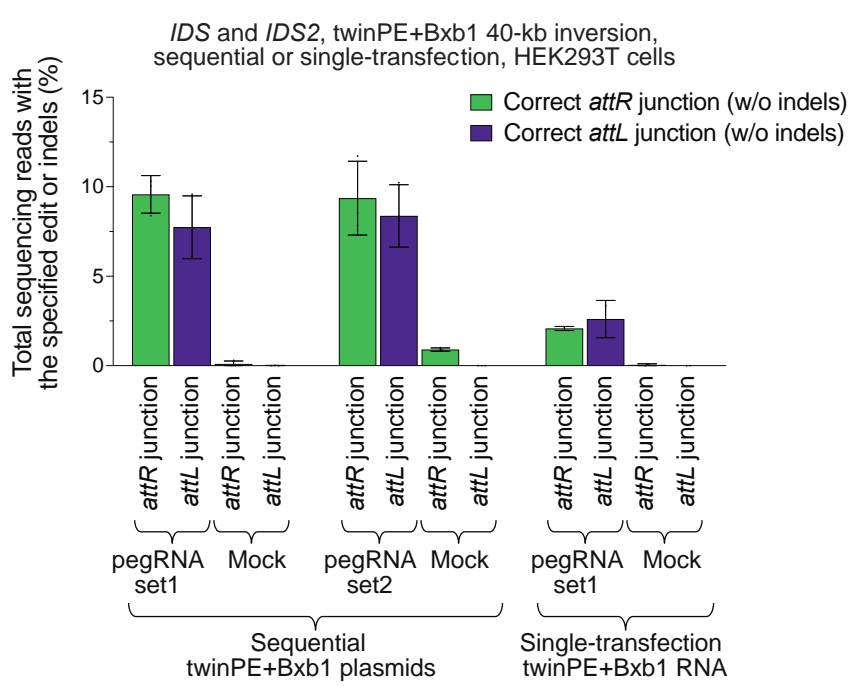

Figure 4 | Site-specific large genomic sequence inversion with twinPE and Bxb1 recombinase in human cells. (a) Schematic diagram of DNA recombination hot spots in $I D S$ and IDS2 that lead to pathogenic 39-kb inversions, and the combined twinPE-Bxb1 strategy for installing or correcting the IDS inversion. (b) Screen of pegRNA pairs at IDS and 
IDS2 for insertion of attP or attB recombination sites. (c) DNA sequencing analysis of the IDS and IDS2 loci after twinPE-mediated insertion of attP or attB sequences, with or without subsequent transfection with Bxb1 recombinase. P-values were derived from a Student's two-tailed $t$-test. (d) 40,167-bp IDS inversion product purities at the anticipated inversion junctions after twinPE-mediated attachment site installation and sequential transfection with Bxb1 recombinase. (e) Analysis of inversion efficiency by amplicon sequencing at IDS and IDS2 loci after sequential transfection or single-step transfection of twinPE editing components and Bxb1 recombinase. Values and error bars reflect the mean and s.d. of three independent biological replicates for (b, $c$ and $d$ ) and two or three independent biological replicates for (e). 


\section{Methods}

General methods. DNA amplification was conducted by PCR using Phusion U Green Multiplex PCR Master Mix (ThermoFisher Scientific) or Q5 Hot Start High-Fidelity 2x Master Mix (New England BioLabs) unless otherwise noted. DNA oligonucleotides were obtained from Integrated DNA Technologies. Plasmids expressing sgRNAs were constructed by ligation of annealed oligonucleotides into BsmBl-digested acceptor vector as previously described ${ }^{12}$.Plasmids expressing pegRNAs were constructed by Gibson assembly or Golden Gate assembly as previously described ${ }^{12}$. Sequences of sgRNA and pegRNA constructs used in this work are listed in Supplementary Table 1. All vectors for mammalian cell experiments were purified using Plasmid Plus Midiprep kits (Qiagen), PureYield plasmid miniprep kits (Promega), or QIAprep Spin Miniprep kits. Synthetic pegRNAs were ordered from IDT without HPLC purification.

General mammalian cell culture conditions. HEK293T (ATCC CRL-3216), U2OS (ATTC HTB-96), K562 (CCL-243), and HeLa (CCL-2) cells were purchased from ATCC and cultured and passaged in Dulbecco's Modified Eagle's Medium (DMEM) plus GlutaMAX

(ThermoFisher Scientific), McCoy's 5A Medium (Gibco), RPMI Medium 1640 plus GlutaMAX (Gibco), or Eagle's Minimal Essential Medium (EMEM, ATCC), respectively, each

supplemented with 10\% (v/v) fetal bovine serum (Gibco, qualified) and $1 \mathrm{x}$

Penicillin+Streptomycin (Corning). All cell types were incubated, maintained, and cultured at $37^{\circ} \mathrm{C}$ with $5 \% \mathrm{CO}_{2}$. Cell lines were authenticated by their respective suppliers and tested negative for mycoplasma.

\section{HEK293T, HeLa, and Huh7 tissue culture transfection protocol and genomic DNA} preparation. HEK293T cells were seeded on 48-well poly-D-lysine coated plates (Corning). 16-24 $\mathrm{h}$ post-seeding, cells were transfected at approximately $60 \%$ confluency with $1 \mu \mathrm{L}$ of Lipofectamine 2000 (Thermo Fisher Scientific) according to the manufacturer's protocols and either: $750 \mathrm{ng}$ of PE2 plasmid DNA, $125 \mathrm{ng}$ of pegRNA 1 plasmid DNA, and $125 \mathrm{ng}$ of pegRNA 2 plasmid DNA (for twinPE transfections); 750 ng of PE2 plasmid DNA, 250 ng of pegRNA plasmid DNA, and 83 ng of sgRNA plasmid DNA (for PE3 transfections); or, 750 ng of Cas9 plasmid DNA and $125 \mathrm{ng}$ of sgRNA 1 plasmid DNA, and $125 \mathrm{ng}$ of sgRNA 2 plasmid DNA (for paired Cas9 nuclease transfections). Unless otherwise stated, cells were cultured 3 days following transfection, after which the media was removed, the cells were washed with 1x PBS solution (Thermo Fisher Scientific), and genomic DNA was extracted by the addition of $150 \mu \mathrm{L}$ of freshly prepared lysis buffer $(10 \mathrm{mM}$ Tris- $\mathrm{HCl}, \mathrm{pH} 7.5 ; 0.05 \%$ SDS; $25 \mu \mathrm{g} / \mathrm{mL}$ Proteinase K (ThermoFisher Scientific)) directly into each well of the tissue culture plate. The genomic DNA mixture was incubated at $37^{\circ} \mathrm{C}$ for $1-2 \mathrm{~h}$, followed by an $80^{\circ} \mathrm{C}$ enzyme inactivation step for $30 \mathrm{~min}$. Primers used for mammalian cell genomic DNA amplification are listed in Supplementary Table 2. For HeLa cell transfections, cells were grown and seeded in 96-well plates (Falcon Catalog \#353075). 16-24 h post-seeding, cells were transfected 0.75 $\mu \mathrm{L}$ of TransIT-HeLaMONSTER transfection reagent (Mirus) using $190 \mathrm{ng}$ of PE2-P2A-Blast plasmid DNA, $31.5 \mathrm{ng}$ of pegRNA 1 plasmid DNA, and $31.5 \mathrm{ng}$ of pegRNA 2 plasmid DNA (for twinPE transfections). $24 \mathrm{~h}$ after transfection, cells were treated with blasticidin to a final concentration of $10 \mu \mathrm{g} / \mathrm{mL}$. Genomic DNA isolation was performed as described above for HEK293T cells using $50 \mu \mathrm{L}$ of lysis buffer. Huh7 cells were seeded at 150,000 cells per well in poly-D-lysine coated 24-well plates (Corning). 16-24 h post-seeding, cells were transfected with $2 \mu \mathrm{L}$ of Lipofectamine 2000 (Thermo Fisher Scientific) according to the manufacturer's protocols and up to $800 \mathrm{ng}$ of plasmid DNA (same ratios as in HEK293T transections 
described above, scaled proportionally). Genomic DNA isolation was performed as described above for HEK293T transfections.

High-throughput DNA sequencing of genomic DNA samples. Genomic sites of interest were amplified from genomic DNA samples and sequenced on an Illumina MiSeq as previously described with the following modifications ${ }^{10}$. Briefly, amplification primers containing Illumina forward and reverse adapters (Supplementary Table 2) were used for a first round of PCR (PCR 1) amplifying the genomic region of interest. 25- $\mu \mathrm{L}$ PCR 1 reactions were performed with $0.5 \mu \mathrm{M}$ of each forward and reverse primer, $1 \mu \mathrm{L}$ of genomic DNA extract and $12.5 \mu \mathrm{L}$ of Phusion $U$ Green Multiplex PCR Master Mix. PCR reactions were carried out as follows: $98{ }^{\circ} \mathrm{C}$ for $2 \mathrm{~min}$, then 30 cycles of $\left[98{ }^{\circ} \mathrm{C}\right.$ for $10 \mathrm{~s}, 61^{\circ} \mathrm{C}$ for $20 \mathrm{~s}$, and $72{ }^{\circ} \mathrm{C}$ for $30 \mathrm{~s}$, followed by a final $72{ }^{\circ} \mathrm{C}$ extension for $2 \mathrm{~min}$. Unique Illumina barcoding primer pairs were added to each sample in a secondary PCR reaction (PCR 2). Specifically, $25 \mu \mathrm{L}$ of a given PCR 2 reaction contained $0.5 \mu \mathrm{M}$ of each unique forward and reverse Illumina barcoding primer pair, $1 \mu \mathrm{L}$ of unpurified PCR 1 reaction mixture, and $12.5 \mu \mathrm{L}$ of Phusion U Green Multiplex PCR 2x Master Mix. The barcoding PCR 2 reactions were carried out as follows: $98^{\circ} \mathrm{C}$ for $2 \mathrm{~min}$, then 12 cycles of $\left[98^{\circ} \mathrm{C}\right.$ for $10 \mathrm{~s}, 61^{\circ} \mathrm{C}$ for $20 \mathrm{~s}$, and $72^{\circ} \mathrm{C}$ for $30 \mathrm{~s}$ ], followed by a final $72{ }^{\circ} \mathrm{C}$ extension for $2 \mathrm{~min}$. PCR products were evaluated analytically by electrophoresis in a 1.5\% agarose gel. PCR 2 products (pooled by common amplicons) were purified by electrophoresis with a 1.5\% agarose gel using a QIAquick Gel Extraction Kit (Qiagen), eluting with $40 \mu \mathrm{L}$ of water. DNA concentration was measured by fluorometric quantification (Qubit, ThermoFisher Scientific) or qPCR (KAPA Library Quantification KitIllumina, KAPA Biosystems) and sequenced on an Illumina MiSeq instrument according to the manufacturer's protocols.

Sequencing reads were demultiplexed using MiSeq Reporter (Illumina). Alignment of amplicon sequences to a reference sequence was performed using CRISPResso2 ${ }^{76}$. For all prime editing yield quantification, prime editing efficiency was calculated as: \% of [\# of reads with the desired edit that do not contain indels] $\div$ [\# of total reads]. For quantification of editing, CRISPResso2 was run in HDR mode using the desired allele as the expected allele (e flag), and with "discard_indel_reads" on. Any sequence containing an indels with respect to the allele to which it aligns was counted separately and did not contribute to the correctly edited allele percentage. The percent editing was quantified as the number of non-discarded reads aligning to the anticipated edited allele (not containing indels) divided by the total number of sequencing reads (which includes those aligned to reference with or without indels and those aligned to the desired edit with or without indels). Indels were quantified as the total number of discarded reads (from either the original or edited allele alignments) divided by the total number of sequencing reads. Editing yield was then calculated as: [\# of nondiscarded HDR aligned reads] $\div$ [total reads]. Indel yields were calculated as: [\# of indelcontaining discarded reads] $\div$ [total reads].

Unique molecular identifiers (UMIs) were applied to quantify the deletion efficiency and assess PCR bias (see Supplementary Note 1 for discussion) in a three-step PCR protocol. Briefly, in the first step of linear amplification, $1 \mu \mathrm{L}$ of genomic DNA extract was linearly amplified by $0.1 \mu \mathrm{M}$ of only the forward primer containing a 15-nt or 16-nt UMI with Phusion $\mathrm{U}$ Green Multiplex PCR Master Mix in a $25-\mu \mathrm{L}$ reaction $\left(10\right.$ cycles of $98^{\circ} \mathrm{C}$ for $1 \mathrm{~min}, 61^{\circ} \mathrm{C}$ for $25 \mathrm{~s}$, and $72{ }^{\circ} \mathrm{C}$ for $\left.1 \mathrm{~min}\right)$. The PCR products were then purified by $1.6 \mathrm{X}$ AmPure beads (Beckman Coulter) and eluted in $20 \mu \mathrm{L}$ of QIAGEN elution buffer. In the second step, 1 or 2 $\mu \mathrm{L}$ of purified linearly amplified PCR products were then amplified for 30 cycles with $0.5 \mu \mathrm{M}$ of each forward and reverse primer with Phusion U Green Multiplex PCR mix in a $25-\mu \mathrm{L}$ reaction as described above. In this case, the forward primer anneals to the P5 Illumina 
adaptor sequence located at the 5' of the UMI primer and upstream of the UMI sequence. For the $D M D$ locus library preparation, the PCR products were purified by $1 \mathrm{X}$ AmPure beads and eluted in $25 \mu \mathrm{L}$ of elution buffer. In the third step, the purified PCR products $(1 \mu \mathrm{L})$ were amplified for 12 cycles as described above for adding unique Illumina barcodes and adaptors. To assess large deletions at the DMD locus, the top band (unedited large amplicon) and bottom band (edited amplicons with deletions) were excised separately from a $1.5 \%$ agarose gel and loaded on two separate MiSeq runs to avoid biased clustering of amplicons. For library preparation at other loci, $1 \mu \mathrm{L}$ of PCR product was used directly for the barcoding PCR step without $1 \mathrm{X}$ AmPure beads purification. For UMI-based PCR bias assessment described in Supplementary Note 1, the editing efficiency was calculated from the libraries prepared following the UMI protocols analyzed with UMI-deduplication or without UMI-deduplication.

Raw sequencing reads were UMI deduplicated using AmpUMI ${ }^{77}$. For paired-end reads, SeqKit ${ }^{78}$ was used to concatenate (merge without overlap) $\mathrm{R} 1 \mathrm{~s}$ with the reverse complement of R2s. The concatenated R1+R2s were UMI deduplicated using the UMI at the 5 ' end of R1. UMI-deduplicated R1s or concatenated R1+R2s were analyzed using CRISPResso2. For analyzing concatenated $\mathrm{R} 1+\mathrm{R} 2 \mathrm{~s}$, an appropriate concatenated reference amplicon sequence was provided to minimize sequencing alignment artifacts due to the concatenation.

Nucleofection of U2OS and K562. Nucleofection was performed in all experiments that used K562 and U2OS cells. 200,000 cells were used per nucleofection. Counted cells were pelleted and washed with PBS, then resuspended in nucleofection solution following the recommendation of Lonza SE Cell Line 4D-Nucleofector Kit. After nucleofection of the cells, the cells were allowed to incubate in the cuvette at room temperature for 10 minutes. After this time, the contents of the cuvette were transferred to a 48 well plate containing pre-warmed $\left(37^{\circ} \mathrm{C}\right)$ media. Genomic DNA was extracted and prepared for Illumina MiSeq preparation as described above.

Single-step twinPE and Bxb1-mediated DNA donor knock-in and inversions. For singlestep knock-in, HEK293T cells were transfected with 500 ng of PE2 plasmid DNA, 50 ng of each pegRNA plasmid DNA (two in total), 200 ng of codon-optimized Bxb1 plasmid DNA, and 200 ng of DNA donor using Lipofectamine 2000 as described above. For multiplex knock-in, HEK293T cells were transfected with $200 \mathrm{ng}$ of PE2 plasmid, $50 \mathrm{ng}$ of each pegRNA plasmid DNA (four in total), 300 ng of Bxb1 plasmid DNA, and 150 ng of each DNA donor (two in total). Donor plasmid sequences can be found in Supplementary Sequences 1. The codonoptimized Bxb1 gene was purchased from Genscript and can be found in Supplementary Sequence 2. It was assembled into a pCMV expression vector using standard molecular cloning techniques.

For inversion experiments, sequential plasmid transfection and single-step mRNA nucleofection were performed. In the sequential plasmid transfection experiment, HEK293T cells were transfected with $750 \mathrm{ng}$ of PE2 plasmid DNA, $62.5 \mathrm{ng}$ of each pegRNA plasmid DNA (four in total) using Lipofectamine 2000 as described above. After three days, cells were detached and plated in 24-well plates, then serially passaged for about seven days. 48-well plates were seeded with 20,000 cells per well and transfected 16-24 h later with Lipofectamine 2000 and 500 ng of BxB1 plasmid DNA. Genomic DNA was extracted and prepared for Illumina MiSeq as described above. For single-step mRNA nucleofection, 200,000 HEK293T cells were nucleofected with 1,000 ng of PE2 mRNA, 30 pmol each of synthetic pegRNAs (4 pegRNAs in total), and $750 \mathrm{ng}$ of BxB1 mRNA using $20 \mu \mathrm{L}$ of Lonza buffer and cells with program $\mathrm{CM}-130$. Cells were recovered with $80 \mu \mathrm{L}$ of pre-warmed (37 
$\left.{ }^{\circ} \mathrm{C}\right)$ media for five minutes. $25-\mu \mathrm{L}$ samples from the nucleofection cuvette were then added to each well of the 48 -well plate and incubated at $37^{\circ} \mathrm{C}$ for 72 hours prior to isolation of genomic DNA as described above.

Droplet digital PCR analysis of knock-in efficiency. Genomic DNA from crude cell lysates was column purified (Zymo) and DNA concentrations were determined by Nanodrop (Thermo Scientific). Droplet digital PCR was used to determine the abundance of an amplicon containing the genome-donor junction in comparison to a reference gene (ACTB). 100-200 ng of DNA was added to a reaction mixture containing ddPCR Supermix for Probes (Bio-Rad, 1863026), HindIII-HF (0.25 units/ $\mu \mathrm{L}$, New England BioLabs, R3104L), ACTB primers and probes (Supplementary Table 3; $900 \mathrm{nM}$ each primer, $250 \mathrm{nM}$ probe) and genome-donor junction primers and probes (Supplementary Table 3; 900 nM each primer, 250 nM probe) according to the manufacturer's protocol. Droplets were generated using a QX200 Manual Droplet Generator (Bio-Rad, 186-4002). Digital droplet PCR was performed as follows: $95^{\circ} \mathrm{C}$ for $10 \mathrm{~min}$, then 50 cycles of $94^{\circ} \mathrm{C}$ for 30 seconds, $58{ }^{\circ} \mathrm{C}$ for $2 \mathrm{~min}$. Following PCR cycles, a final incubation was conducted at $98^{\circ} \mathrm{C}$ for $10 \mathrm{~min}$. Droplets were read by a QX200 Droplet Reader (Bio-Rad, 1864001) and data were analyzed using QuantaSoft (Bio-Rad).

FIX expression from ALB locus in Huh7 cells. Huh7 cells were transfected with 160 ng of Bxb1 plasmid DNA, $160 \mathrm{ng}$ of donor plasmid DNA (attP-splice acceptor-cDNA of F9 exons 28), $400 \mathrm{ng}$ of prime editor plasmid DNA, and pegRNAs plasmid DNA for installation of attB in the first intron of $A L B$ or at CCR5 (40 ng each). Three days post-transfection, cells were passaged and allowed to grow to confluence. Their media was changed, and they were left to condition the fresh media, with aliquots taken at days 4, 7, and 10. Factor IX concentration in conditioned media was measured by ELISA (Innovative Research Human Total Factor IX ELISA Kit, IHUFIXKTT).

\section{Lentivirus production, GFP reporter cell line construction, and flow cytometry}

analysis. The lentiviral transfer vector described in Extended Data Figure 9 and Supplementary Sequence 3 was assembled using standard molecular cloning techniques. The lentivirus was produced as previously described ${ }^{79,80}$. Briefly, a T-75 flask of rapidly dividing HEK293T cells (ATCC; Manassas, VA, USA) were transfected at 80-90\% confluence with lentivirus helper plasmids pVSV-G and psPAX2 in along with the constructed lentiviral transfer vector using FuGENE HD (Promega) according to the manufacturer's protocol. After 48 hours, supernatant was collected, centrifuged at 3,000 x $g$ for 15 minutes to remove cellular debris, and filtered using a $0.45-\mu \mathrm{m}$ PVDF filter. Filtered supernatant was concentrated using the PEG-it Virus Precipitation Solution (System Biosciences) according to the manufacturer's instructions. The resulting pallet was resuspended in Opti-MEM (Thermo Fisher Scientific) using $1 \%$ of the original medium volume. Resuspended pellet was used to transduce the HEK293T cells with multiplicity of infection. Successfully transduced cells that had stable GFP reporter integration were selected with $2.5 \mu \mathrm{g} / \mathrm{mL}$ puromycin for more than 3 passages and then seeded into 48-well plates for transfection with PE2 plasmid DNA (750 ng), four AAVS1-targeting pegRNAs (62.5 ng each) for multiplexed attP and attB insertion, and Bxb1 plasmid DNA (100 ng, $200 \mathrm{ng}, 500 \mathrm{ng}$, or $1000 \mathrm{ng}$ ) in a single transfection step. Cells were then collected after three days and analyzed by CytoFLEX S Flow Cytometer (Beckman Coulter) and FlowJo v10.

\section{Production of PE2 and Bxb1 mRNA.}


The mRNA was produced using the previously reported protocol ${ }^{41,81}$. Briefly, the PE2 editor and codon optimized Bxb1 coding sequences were cloned into a plasmid encoding a mutated T7 promoter that cannot initiate with mononucleotides. This plasmid was used as template in a PCR reaction (PhusionU 2X Master Mix) using PAGE-purified primers (IDT) Forward: 5'TCGAGCTCGGTACCTAATACGACTCACTATAAGG-3' and Reverse: 5'-

TTTTTTTTTTTTTTTTTTTTTTTTTTTTTTTTTTTTTTTTTTTTTTTTTTTTTTTTTTTTTTTTT TTTTTTTTTTTTTTTTTTTTTTTTTTTTTTTTTTTTTTTTTTTTTTTTTTTTTTTTTTTCTTCCTAC TCAGGCTTTATTCAAAGACCA-3', and the resulting PCR product was purified and used as template for in vitro transcription. The HiScribe T7 High-Yield RNA Synthesis Kit (New England BioLabs) was used according to the company's recommended instruction but with full substitution of N1-methyl-pseudouridine for uridine and co-transcriptional capping with CleanCap AG (TriLink Biotechnologies). RNA was precipitated with lithium chloride (2.5 M final concentration), the pellet was washed with ice cold $70 \%$ ethanol, and then resuspended in water.

\section{Data availability}

High-throughput sequencing data have been deposited to the NCBI Sequence Read Archive database under accession PRJNA770428. 


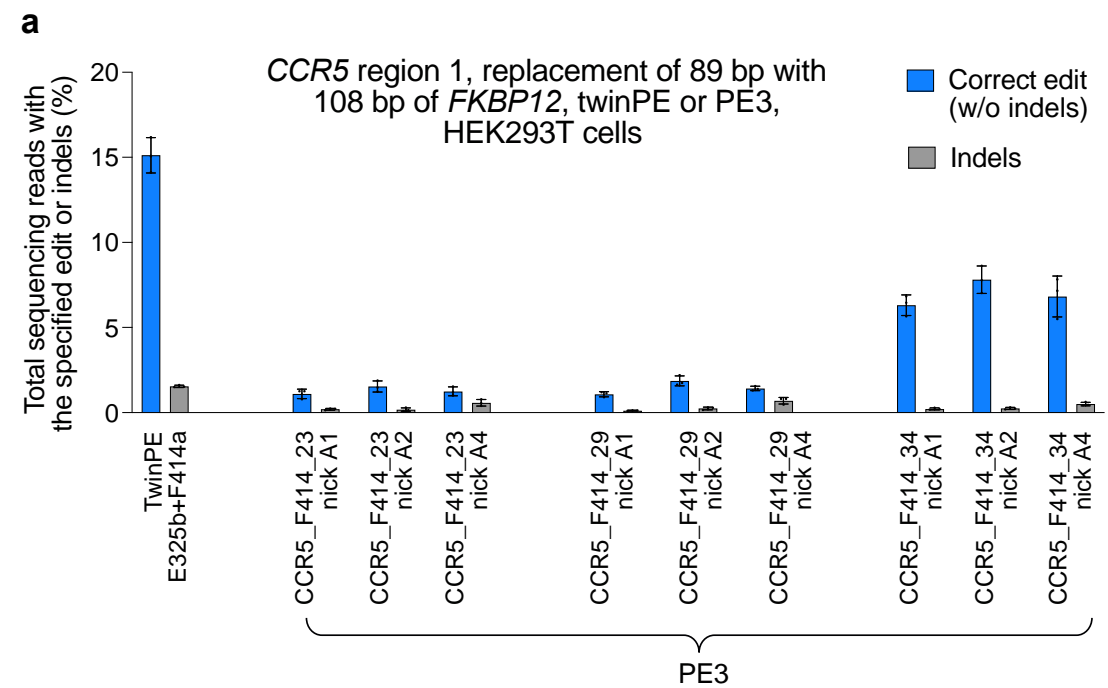

b

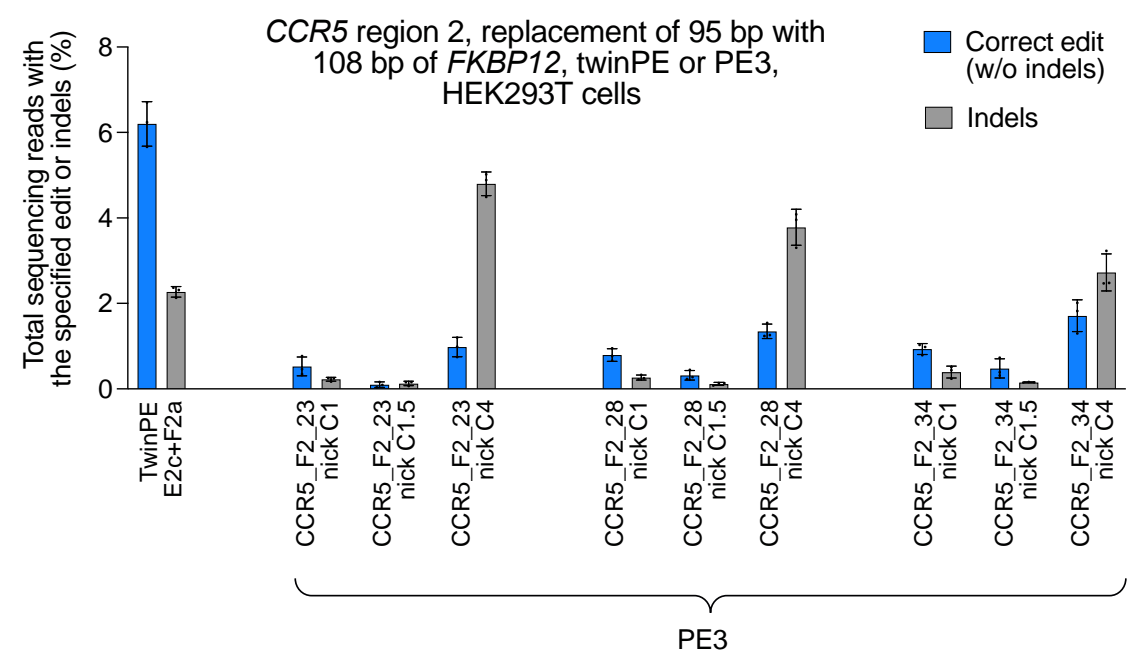

C

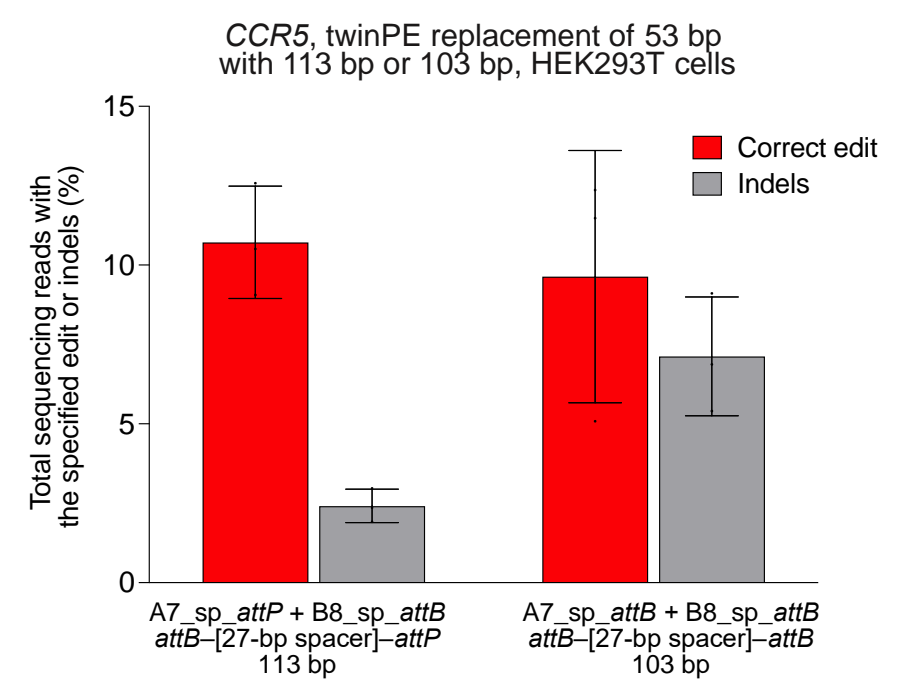

Extended Data Figure 1 | Twin prime editing mediates sequence replacements at CCR5. (a) Replacement of endogenous sequence within CCR5 region 1 with a 108-bp fragment of $F K B P 12 \mathrm{cDNA}$ using twinPE (FKBP12 sequence oriented in the forward direction,) or PE3 (FKBP12 sequence oriented in the reverse direction). For PE3 editing, 
pegRNA RT templates were designed to encode 108 base pairs of FKBP12 cDNA sequence and one of three different target-site homology sequence lengths. For PE3 edits, each pegRNA was tested with three nicking sgRNAs. (b) Replacement of endogenous sequence within CCR5 region 2 with a 108-bp fragment of FKBP12 cDNA sequence using twinPE (FKBP12 sequence oriented in the forward direction) or PE3 (FKBP12 sequence oriented in the reverse direction). As in (a), PE3 edits were tested with pegRNAs containing RT templates that were designed to encode 108 base pairs of FKBP12 cDNA sequence and one of three different target-site homology sequence lengths. For PE3 edits, each pegRNA was tested with three nicking sgRNAs. Values and error bars reflect the mean and s.d. of three independent biological replicates. (c) Transfection of HEK293T cells with a pair of pegRNAs targeting CCR5 leads to replacement of 53 base pairs of endogenous sequence with 113 base pairs (attB-[27-bp spacer]-attP) or 103 base pairs (attB-[27-bp spacer]-attB) of exogenous sequence. Values and error bars reflect the mean and s.d. of three independent biological replicates. 


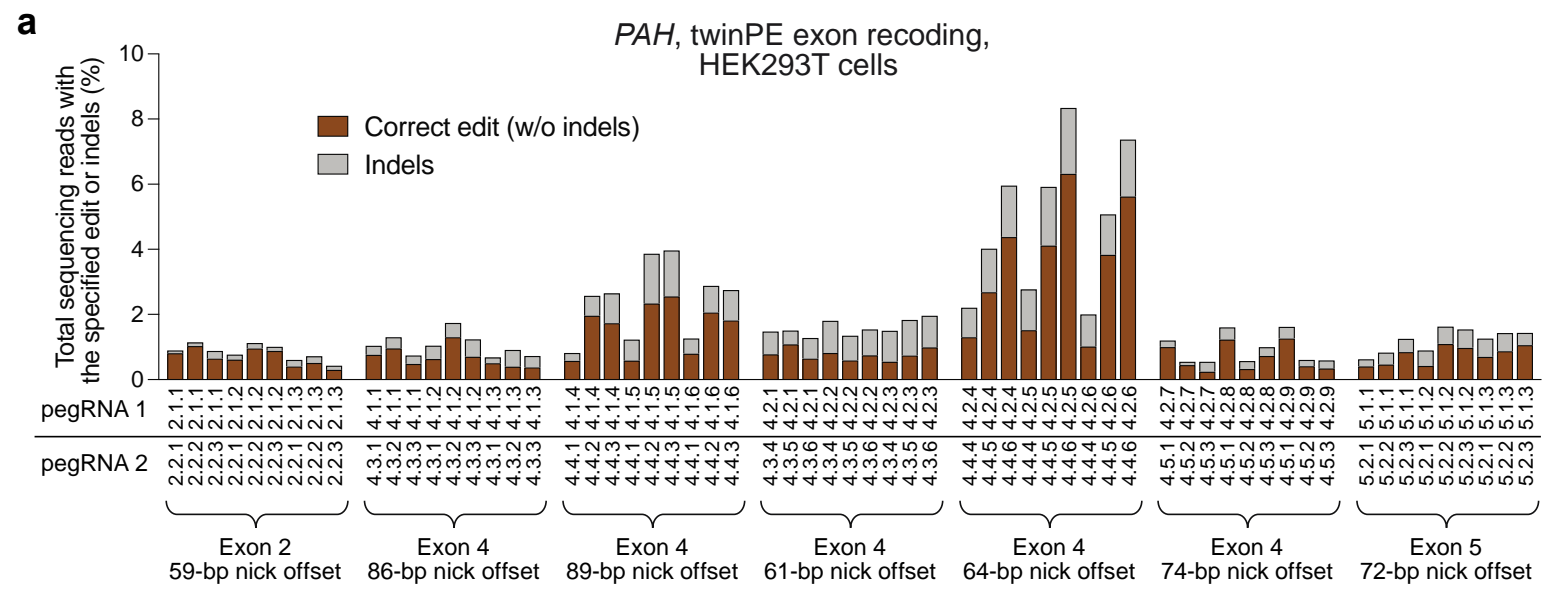

b

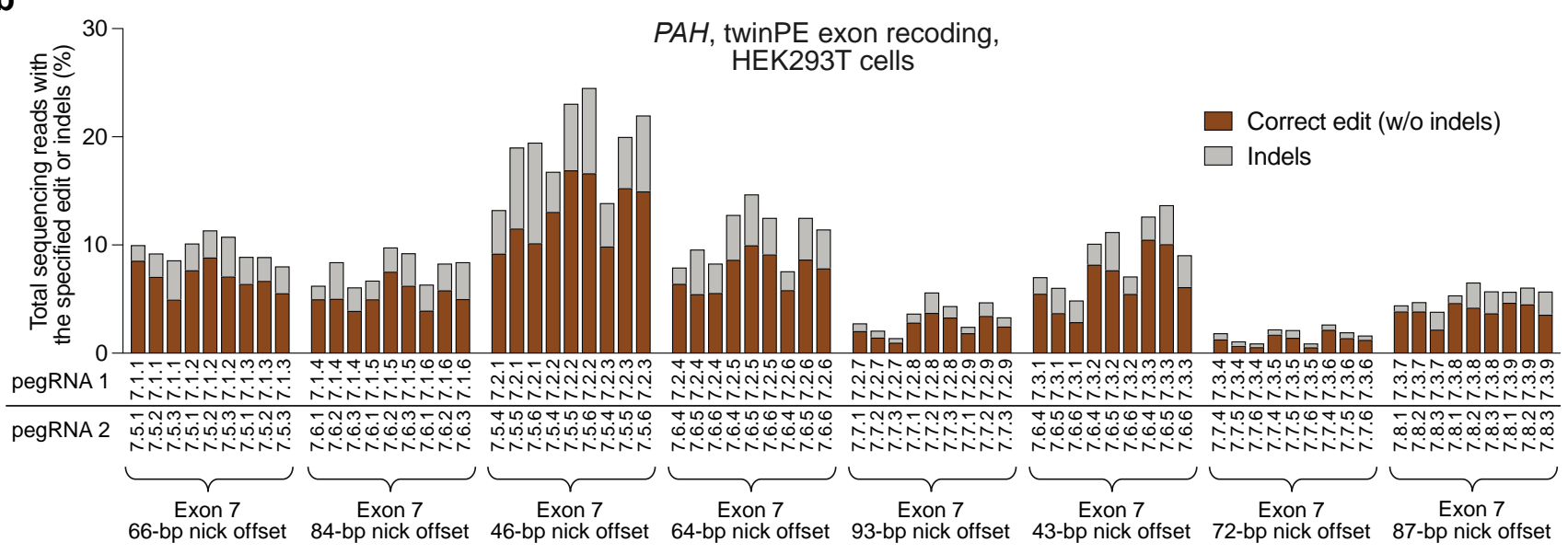

C

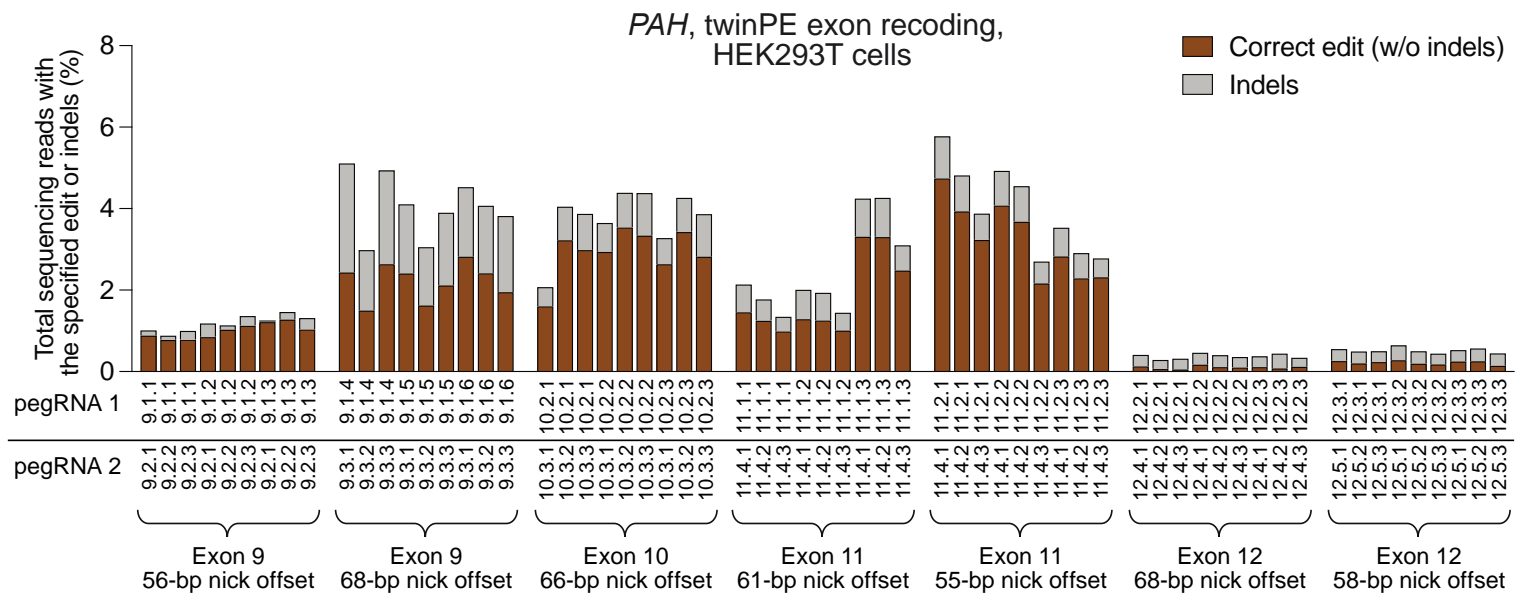

Extended Data Figure 2 | Recoding of PAH exon sequences in HEK293T cells via twinPE. Screen of pegRNA pairs targeting $P A H$ for recoding of (a) exons 2, 4 and 5, (b) exon 7 , and (c) exons $9,10,11$, and 12. RT templates of pegRNAs encoded partially recoded exonic sequence to optimize orthogonality to the endogenous gene sequence. For each spacer pair, nine pegRNA combinations were tested using three PBS variants for each spacer in a three-by-three matrix, with RT templates encoding the recoded exonic sequence, which was held constant for given spacer pairs. Sequences of pegRNAs are listed in Supplementary Table 1. Sequences of recoded exonic sequences are listed in Supplementary Table 4. Values in (a), (b) and exon 9 in (c) reflect single biological 
bioRxiv preprint doi: https://doi.org/10.1101/2021.11.01.466790; this version posted November 2, 2021. The copyright holder for this preprint (which was not certified by peer review) is the author/funder, who has granted bioRxiv a license to display the preprint in perpetuity. It is made available under aCC-BY-NC 4.0 International license.

replicates. Values for exons 10,11 and 12 in (c) reflect the mean of three independent biological replicates. 


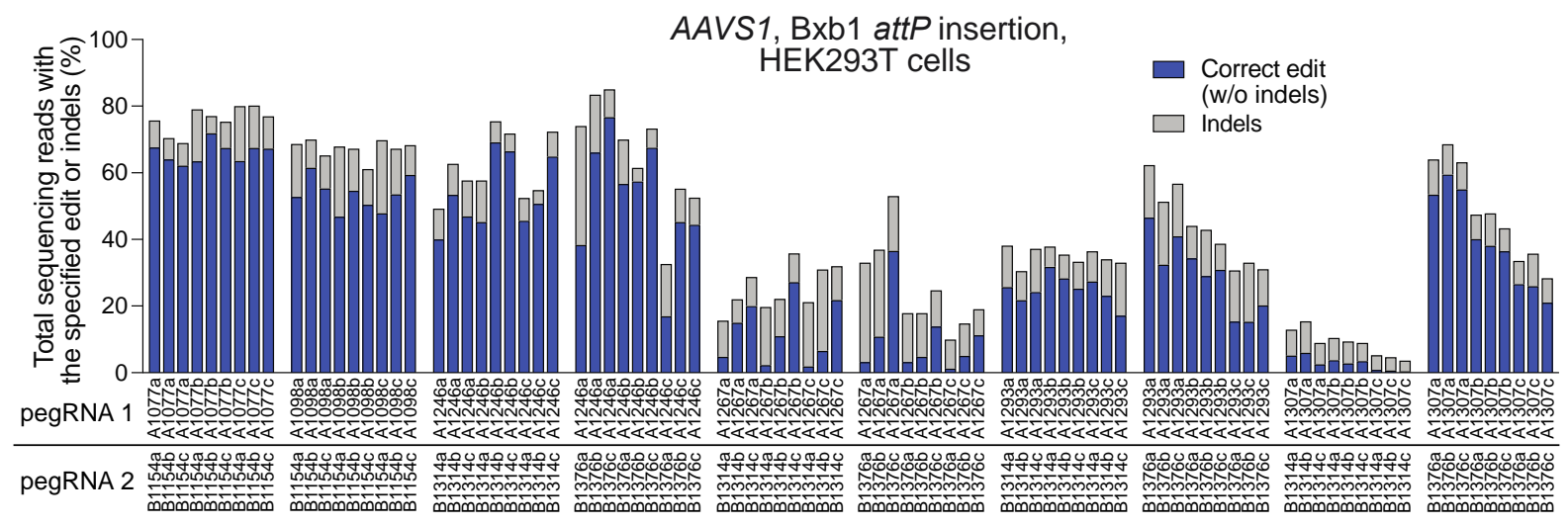

AAVS1, Bxb1 attP insertion,

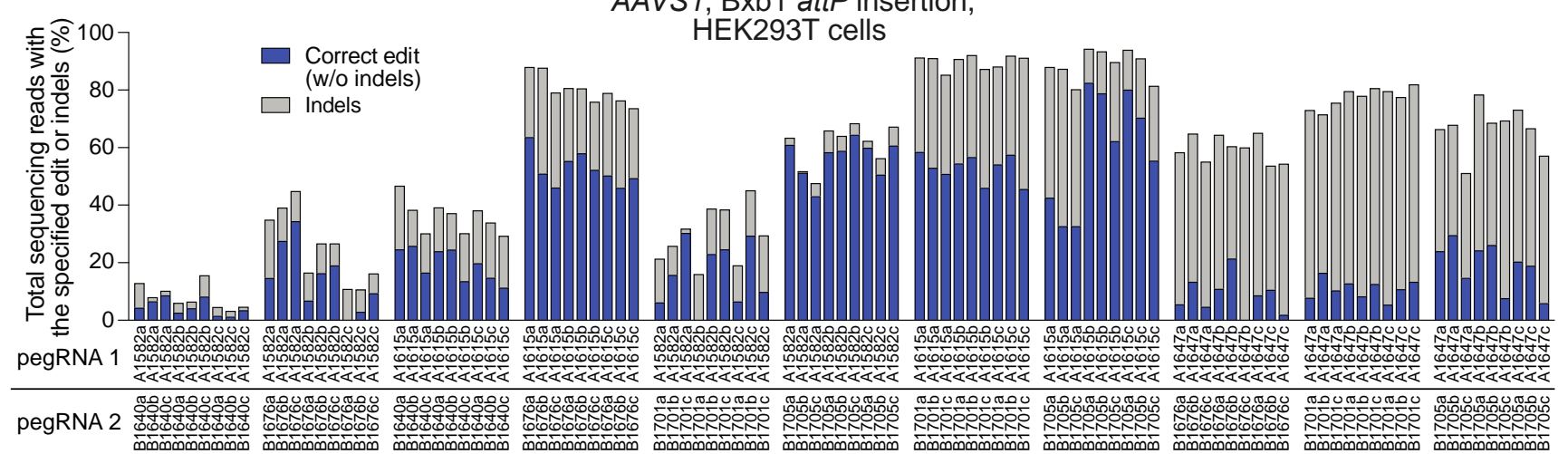

AAVS1, Bxb1 attP insertion,

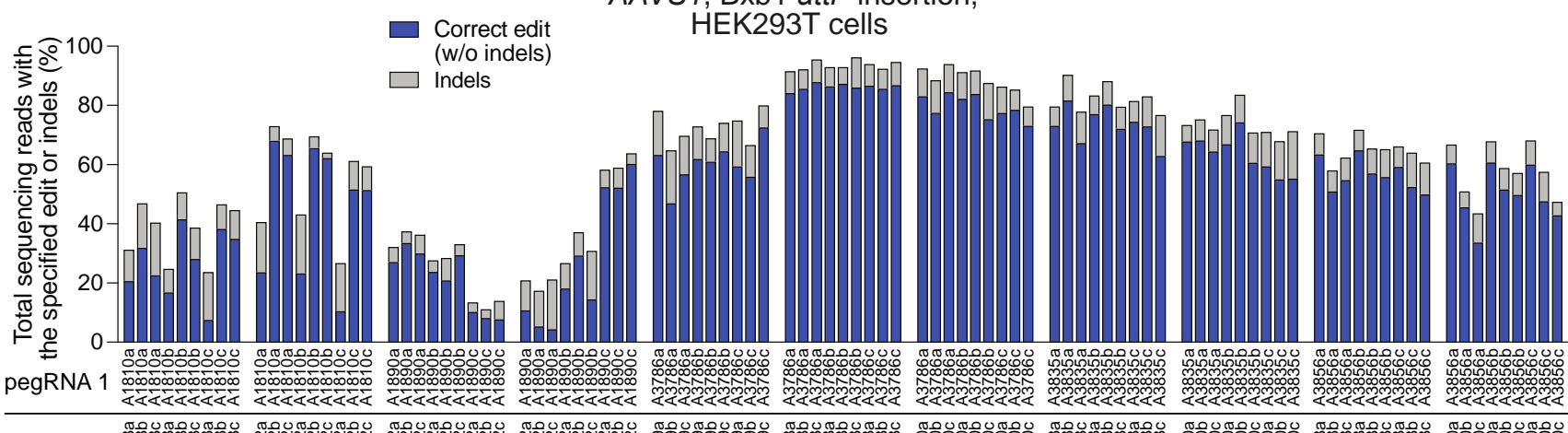

pegRNA 2

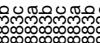

Extended Data Figure 3 | Installation of a 50-bp Bxb1 attP site at AAVS1 with twinPE. Spacer pairs targeting the AAVS1 locus were designed for twinPE-mediated insertion of the Bxb1 attP attachment site. For each spacer, three pegRNAs were designed having three different PBS lengths and a fixed RT template that encodes a portion (43-44 bp) of the Bxb1 attP sequence. Sequences of pegRNAs are listed in Supplementary Table 1. For each spacer pair, a three-by-three matrix of pegRNA combinations was tested by plasmid DNA cotransfection with PE2 in HEK293T cells. Each pegRNA pair is specified below the x-axis. Values reflect single biological replicates. 

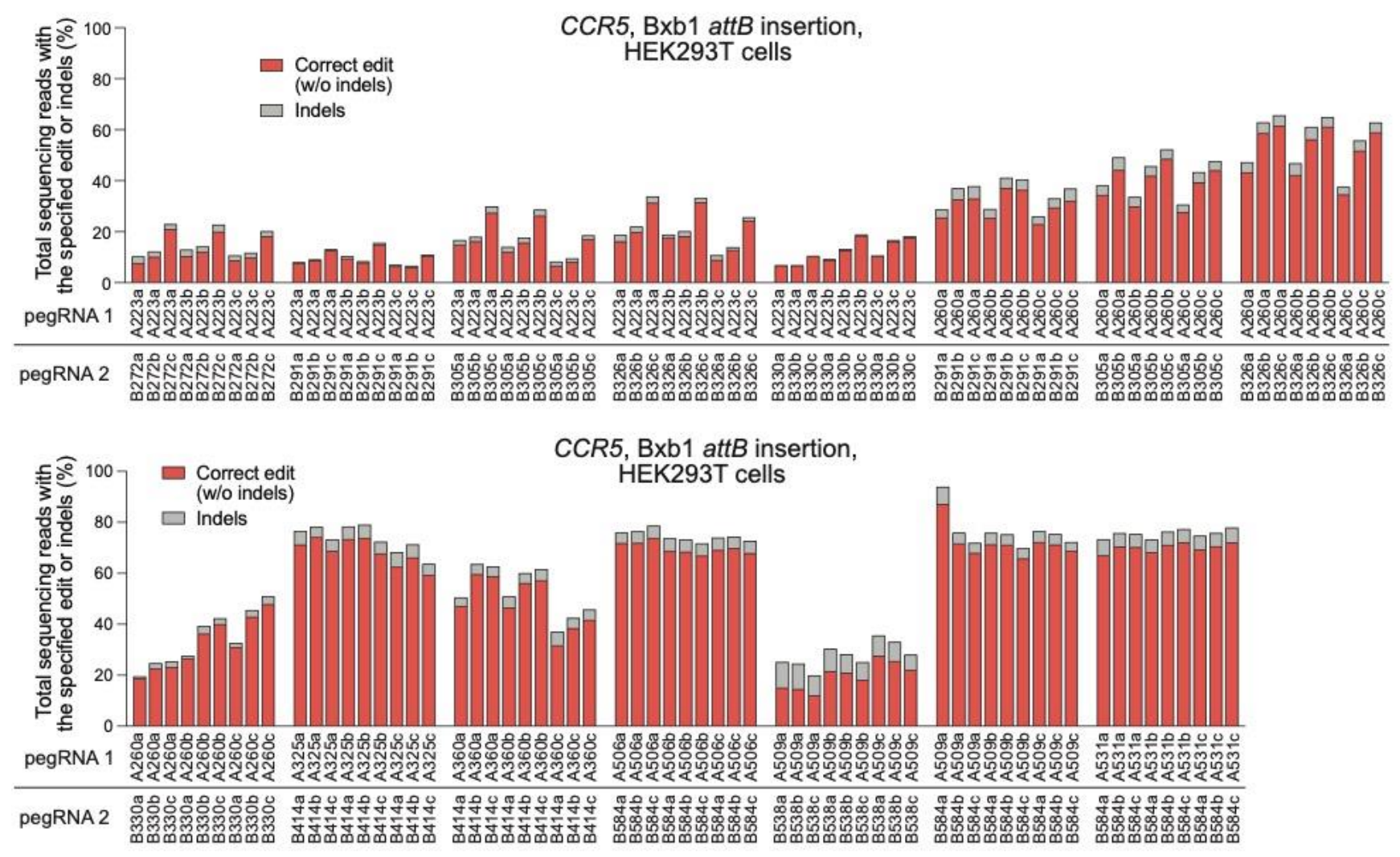

Extended Data Figure 4 | Installation of a 38-bp Bxb1 attB site at CCR5 with twinPE. Spacer pairs targeting the CCR5 locus were designed for twinPE-mediated insertion of the Bxb1 attB attachment site. For each spacer, three pegRNAs were designed having three different PBS lengths and a fixed RT template that encodes the full-length Bxb1 attB sequence (38 bp). Sequences of pegRNAs are listed in Supplementary Table 1. For each spacer pair, a three-by-three matrix of pegRNA combinations was tested by plasmid DNA cotransfection with PE2 in HEK293T cells. Each pegRNA pair is specified below the $x$-axis. Values reflect single biological replicates. 
a

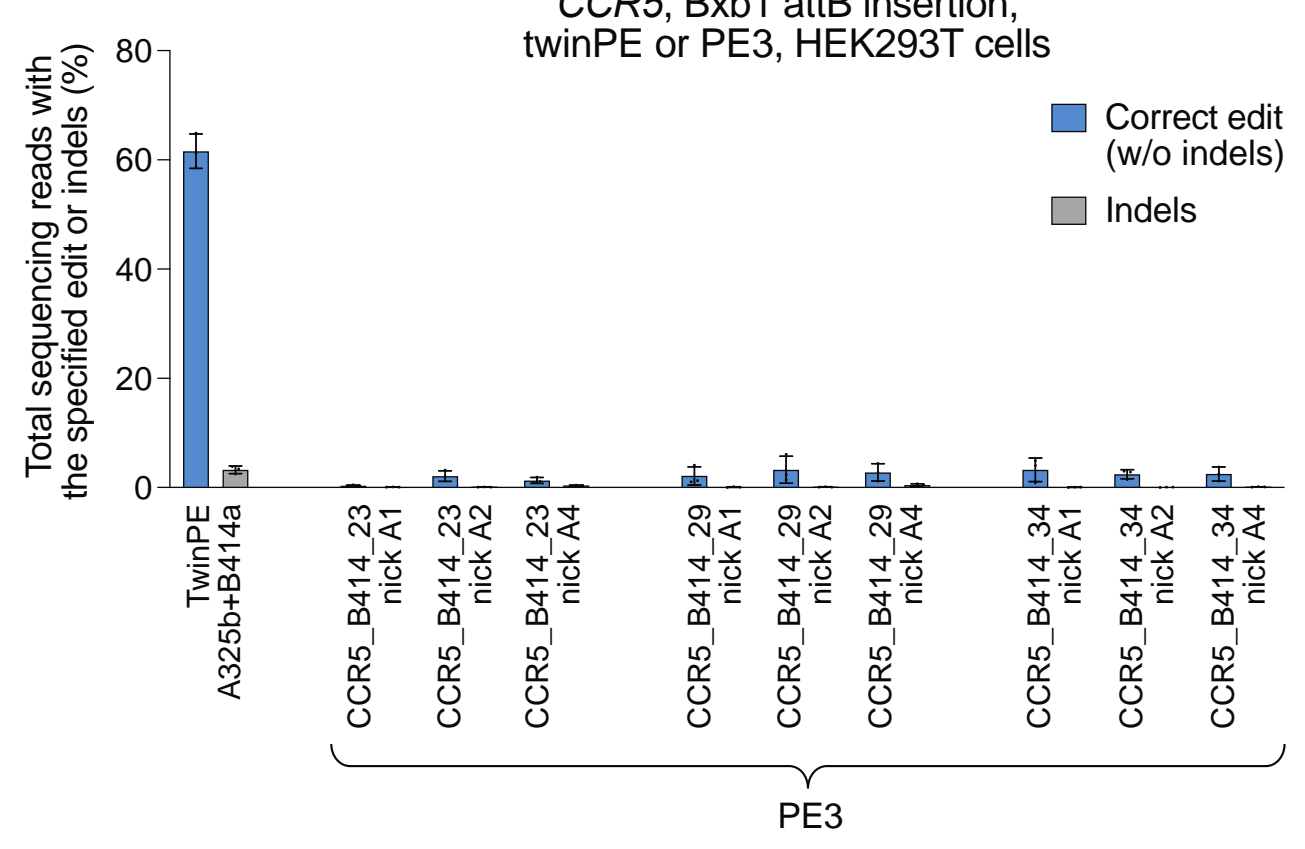

b

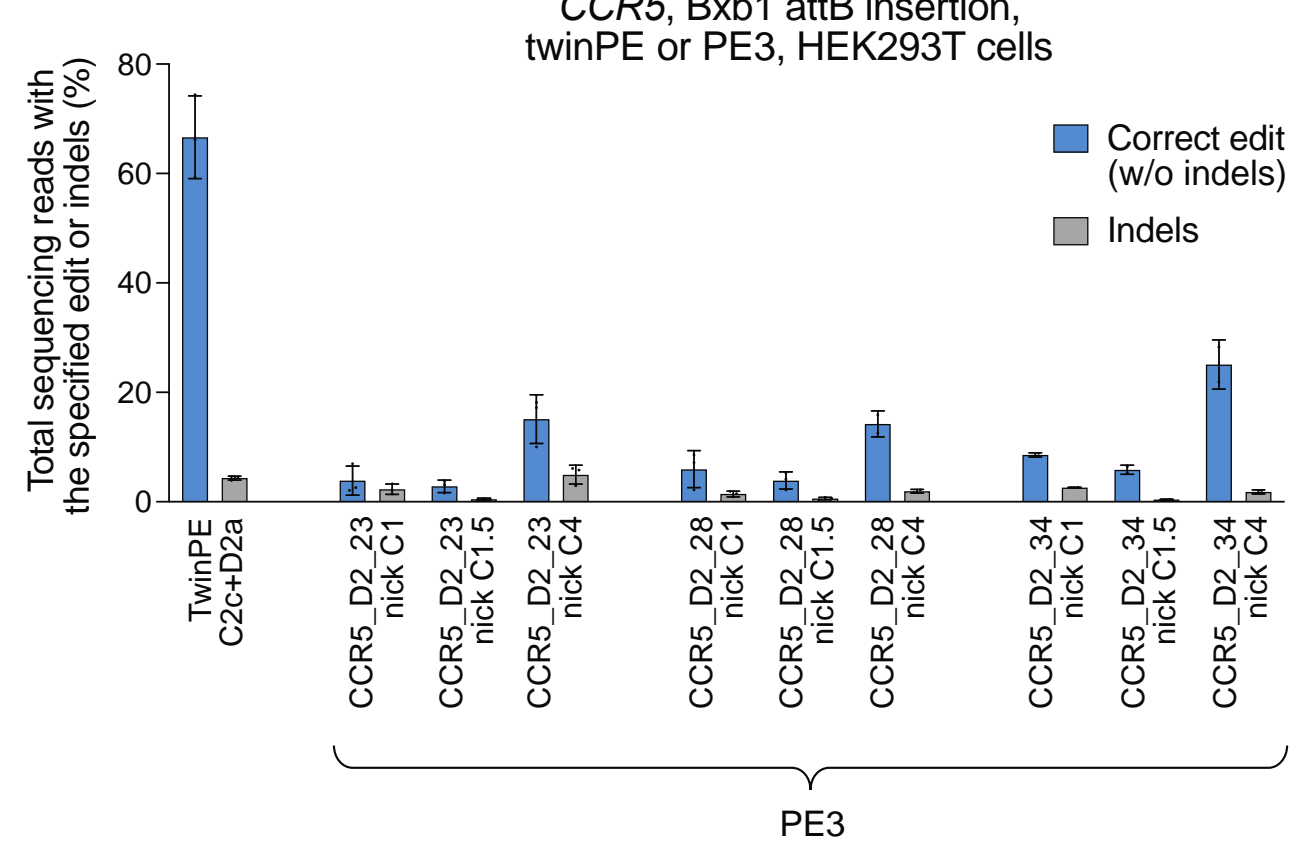

Extended Data Figure 5 | Comparison of twinPE and PE3 for Bxb1 attB insertion at CCR5. (a) Replacement of endogenous sequence within CCR5 region 1 with the Bxb1 attB site using twinPE or PE3. For PE3 editing systems, pegRNA RT templates were designed to encode the Bxb1 attB sequence and one of three different target-site homology sequence lengths. For PE3 edits, each pegRNA was tested with three nicking sgRNAs. (b) Replacement of endogenous sequence within CCR5 region 2 with the Bxb1 attB sequence using twinPE or PE3. As in (a), PE3 edits were tested with pegRNAs containing RT templates that were designed to encode the Bxb1 attB sequence and one of three different target-site homology sequence lengths and tested with three nicking sgRNAs. Values and error bars reflect the mean and s.d. of three independent biological replicates. 
a
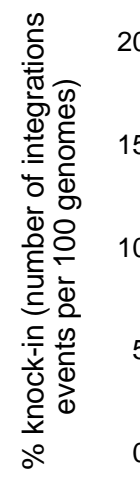

20
5
10
5
0

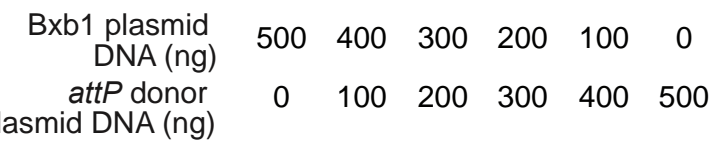

C

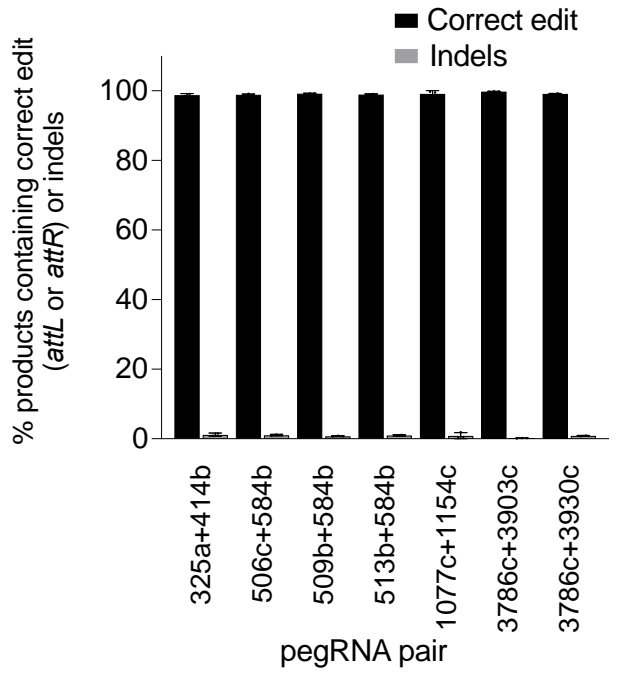

e

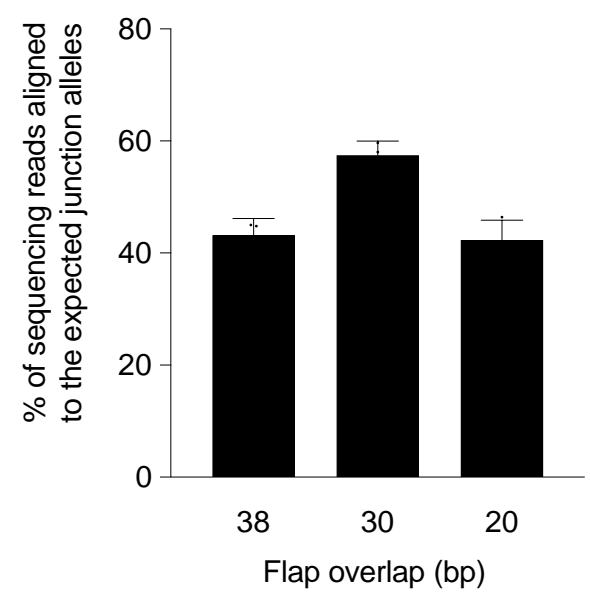

b

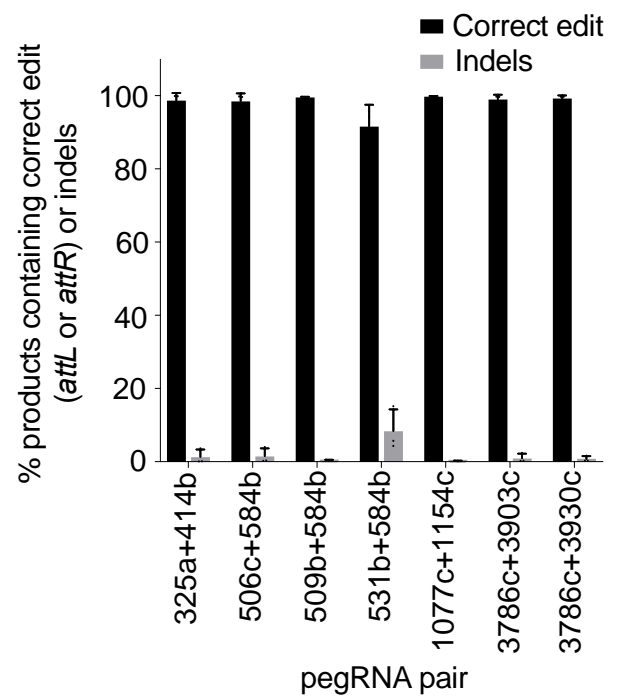

d

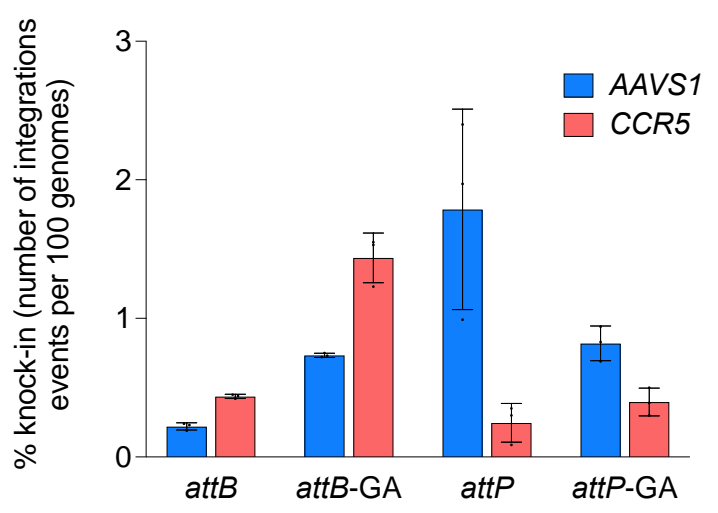

$\mathbf{f}$

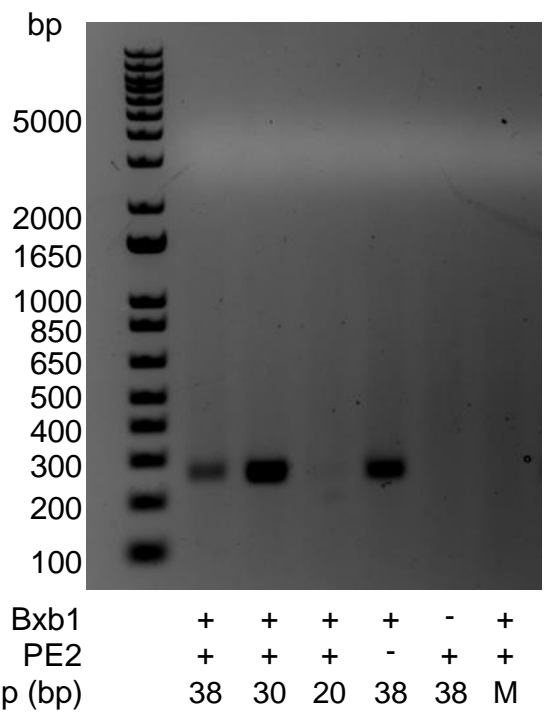

Extended Data Figure 6 | TwinPE combined with Bxb1 recombinase for targeted knockin of donor DNA plasmids. (a) Bxb1-mediated DNA donor knock-in in clonal HEK293T cell lines. Transfection of a HEK293T clonal cell line containing homozygous attB site insertion at 
CCR5 with varying amounts of Bxb1-expressing plasmid and attP-containing donor DNA plasmid. Knock-in efficiency was quantified by ddPCR. Values and error bars reflect the mean and s.d. of two independent biological replicates. (b) Assessment of genome-donor junction purity by high-throughput sequencing. Genomic DNA from single-transfection knockin experiments was amplified with a forward primer that binds the genome and a reverse primer that binds within the donor plasmid (Supplementary Table 2). Values and error bars reflect the mean and s.d. of three independent biological replicates. (c) Assessment of genome-donor junction purity at the other junction by high-throughput sequencing as performed in (b). (d) Multiplexed single-transfection knock-in at AAVS1 and CCR5. HEK293T cells were transfected with plasmids encoding PE2, Bxb1, a pair of pegRNAs for the insertion of attP at $A A V S 1$, an attB-donor, a pegRNA pair for the insertion of one of four attachment sites (attB, attB-GA, attP, or attP-GA) at CCR5, and a corresponding donor. Knock-in was observed at both target loci under all four conditions. Insertion of attP at $A A V S 1$ and attB at CCR5 gave the lowest knock-in efficiencies overall (0.2\% at AAVS1, $0.4 \%$ at CCR5). Insertion of attP at both sites yielded the highest levels of knock-in at AAVS1 (1.8\%) but low levels $(0.2 \%)$ at CCR5. When an orthogonal edit (attB-GA or attP-GA) was introduced at CCR5, AAVS1 knock-in was 0.7-0.8\%. Higher knock-in at CCR5 was observed with attB-GA $(1.4 \%)$ than with attP-GA $(0.4 \%)$, consistent with our single locus knock-in results. Values and error bars reflect the mean and s.d. of three independent biological replicates. (e) and (f) Effects of reducing pegRNA overlap on twinPE efficiency and donor/pegRNA recombination.

(e) The editing efficiencies of pairs of pegRNAs for insertion of Bxb1 attB at CCR5 were measured by high-throughput sequencing. The pairs differed in the amount of overlap shared between their flaps, from $38 \mathrm{bp}$ (full-length attB sequence) down to $20 \mathrm{bp}$. Editing efficiency of the pairs with shorter overlaps was comparable to the pair with full-length overlap. Values and error bars reflect the mean and s.d. of three independent biological replicates. (f) Assessment of recombination between attB-containing pegRNA plasmids and attP-containing donor plasmids. Following transfection of HEK293T cells with the indicated samples, isolated DNA was amplified with a forward primer that binds the pegRNA expression plasmid (TTGAAAAAGTGGCACCGAGT) and a reverse primer that binds the donor plasmid (CTCCCACTCATGATCTA). A positive 256-bp PCR band confirms recombination between the two plasmids. When the pegRNA encodes full-length attB (38-bp) or a truncated version of attB with 30-bp of overlap between flaps, a band is observed; however, recombination is not observed when the pegRNAs encode a truncated attB with only 20-bp of flap overlap. The "No PE2" control uses the 38-bp overlap pegRNA pair. No recombination is observed in the absence of Bxb1 or if the donor and pegRNA plasmids both bear attB (Mismatch, "M"). 
a

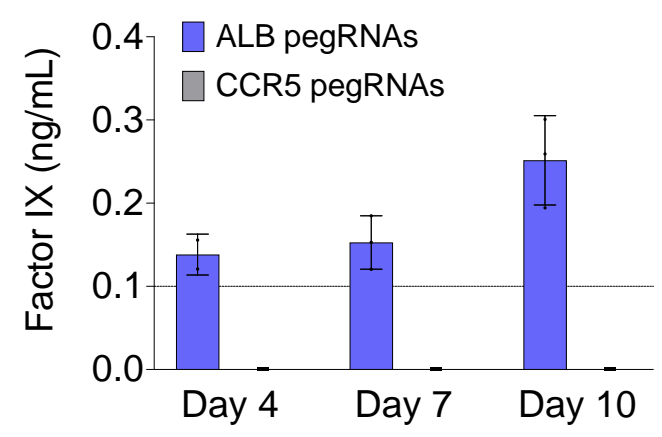

b

\begin{tabular}{|c|c|c|c|c|c|c|c|c|}
\hline & & \multicolumn{4}{|c|}{;062. !. I, \#\$7. 6!\#\$,0!!" " \#\$! } & \multicolumn{3}{|c|}{ 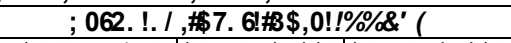 } \\
\hline & 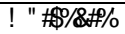 & ()$^{*} \&+,-$, & ${ }^{*} / 01+{ }^{*} 2$ & ${ }^{*} / 3 .+{ }^{*} 2,$. & ${ }^{\star}\left(-.+{ }^{\star} 2\right.$ & $-/ 441+-{ }^{*}, 1$ & $(4201+(3)(1)$ & $(4201+(3 / / 1)$ \\
\hline 5" 6+स $\$ 7 \%$ & ;/ & -40 &, 84 & $0<1$ & $(<4$ & $(24$ & 04( & $0 \ll 44$ \\
\hline $5=$ =61R $\$ 7 / \%$ / & $; / \ll$ & $; / \leqslant$ & $; / \ll$ & $; / \ll$ & $; / \leqslant$ & $; / \leqslant$ & $; / \ll$ & $; / \ll$ \\
\hline $5=6+187 \%$ \% $189: \# / 2$ & $; / \ll$ & $; /<$ & $; /<$ & $; / \ll$ & $; / \ll$ & $; / \ll$ & $; / \ll$ & $; / \ll$ \\
\hline $5=6 \#$ $\$ 7 \% / 289: \# / 2$ & $; / \leqslant$ & $; /<$ & $; / \leqslant$ & $; / \ll$ & $; / \ll$ & $; / \leqslant$ & $; / \ll$ & $; / \leqslant$ \\
\hline $5=6+1 / \$ 7 / \% 99: \# / 8$ & $; / \ll$ & $/ \ll-4$ & $; / \ll$ & $; / \ll$ & $; / \ll$ & $; / \ll$ & $; / \leqslant$ & $1 \ll,-$ \\
\hline $5=6+1 \times \$ 7 \%$ \% & $; / \leqslant$ & $; / \leqslant$ & $; /<$ & $1 \leqslant{ }^{*} 4$ & $; / \leqslant$ & $; / \leqslant$ & $; / \leqslant$ & $; / \leqslant$ \\
\hline
\end{tabular}

\section{C}

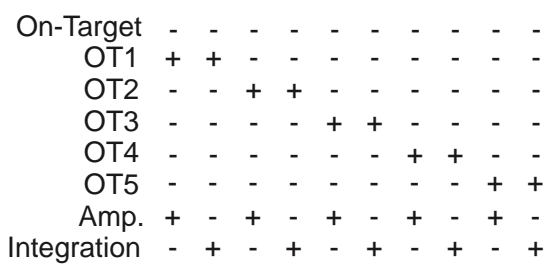

$325 a+414 b$
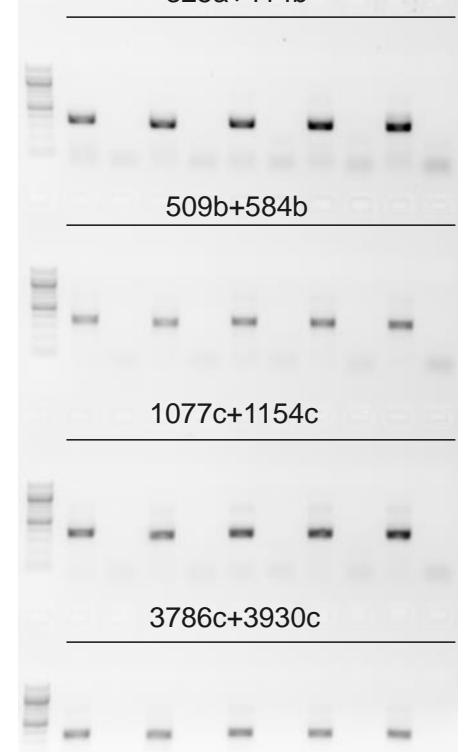

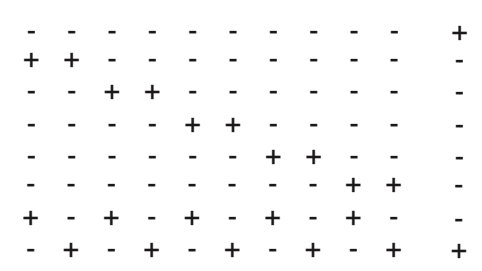

pos.

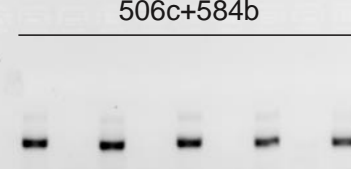

$531 b+584 b$

pos.

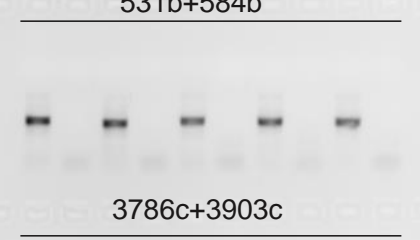

pos.

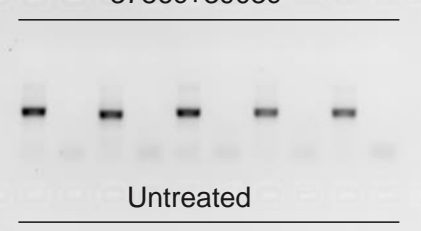

pos.

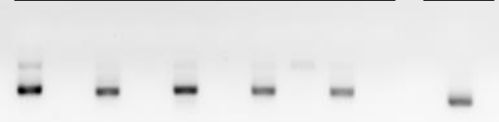

Extended Data Figure 7 | Expression of human Factor IX from the ALB promoter following twinPE-recombinase knock-in and characterization of Bxb1 off-target editing. (a) Huh7 cells were transfected with Bxb1, donor (attP-splice acceptor-cDNA of F9 exons 2- 
8), PE2, and pegRNAs for installation of attB in the first intron of $A L B$ or at CCR5. Three days post-transfection, cells were split and allowed to grow to confluence. Their media was changed, and they were left to condition the fresh media, with aliquots taken at days 4,7 , and 10. Factor IX was present at detectable levels by ELISA (dashed line represents the lower limit of detection) in two of three samples treated with $A L B$ pegRNAs at Day 4, and in all samples treated with $A L B$ pegRNAs at Day 7 and Day 10. Factor IX was never detected in the conditioned media of any samples treated with CCR5 pegRNAs. Values and error bars reflect the mean and s.d. of two or three independent biological replicates. (b) Targeted amplicon sequencing was performed for each of the five nominated pseudo-sites (OT1-OT5) from seven different samples treated with 5.6-kb donor DNA plasmid, twinPE reagents targeting CCR5 or AAVS1, and Bxb1 recombinase. The indels in all five pseudo-sites are either below the limit of detection $(<0.1 \%)$ or near-background compared to untreated controls. (c) To capture potential donor plasmid integration events at nominated pseudosites, primers were used to amplify predicted integration junctions. The gel depicts PCR reactions performed for each off-target site as indicated in the above legend. Confirmation of on-target donor integration from the samples is shown in the right-most column of the gel. In (b) and (c), two or three independent biological replicates were performed. 

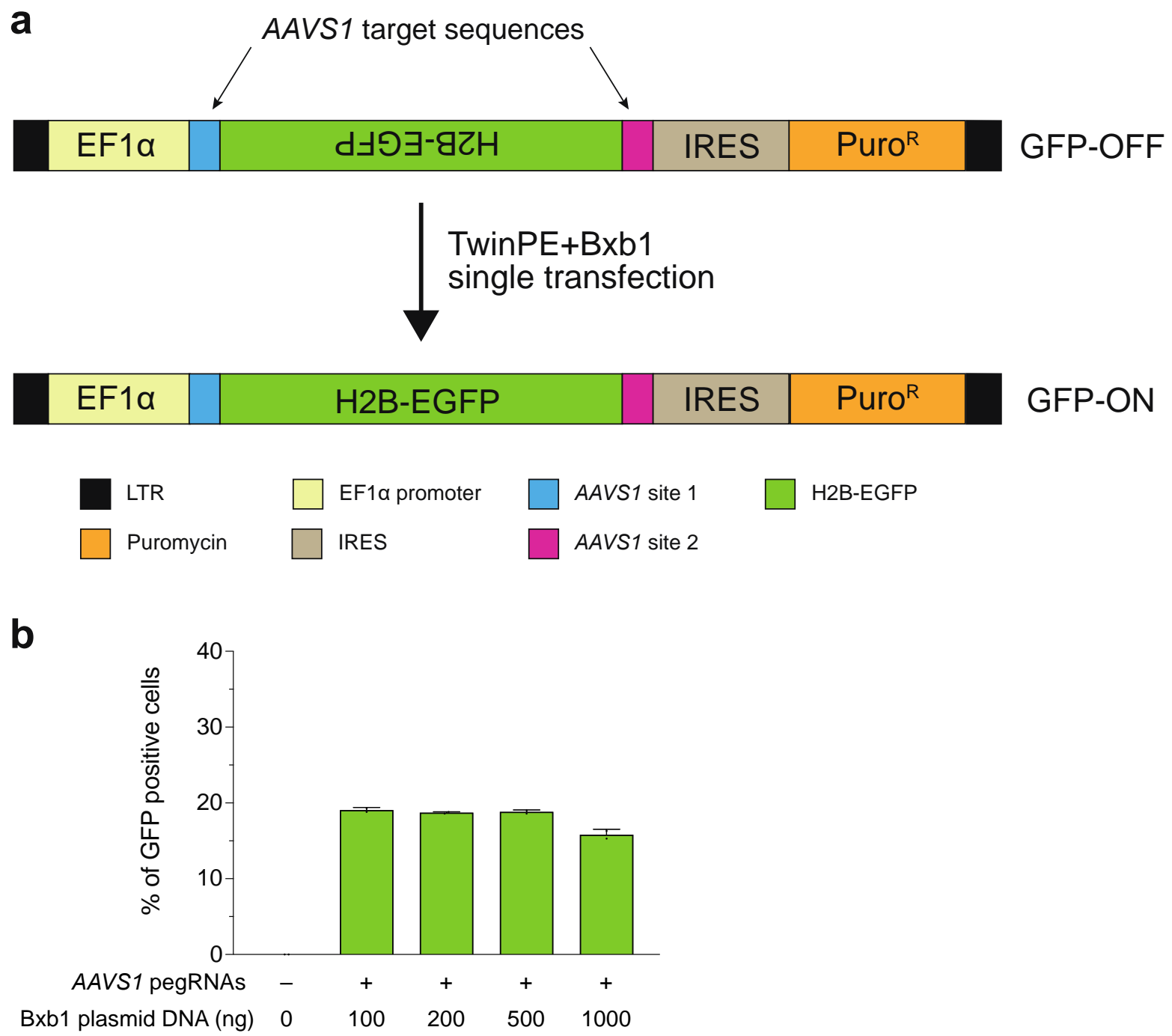

\section{Extended Data Fig. 8 | TwinPE and Bxb1-mediated inversion in HEK293T GFP reporter} cells. (a) The lentiviral fluorescent reporter construct used to assess inversion efficiency with twinPE and Bxb1 recombinase. The reporter contains an EF1a promoter followed by an inverted H2B-EGFP coding sequence that is flanked by partial AAVS1 DNA sequence, an internal ribosome entry site (IRES), and a puromycin resistance gene. Successful installation of opposite-facing attB (left) and attP (right) sequences at the AAVS1 target sequences and subsequent inversion by Bxb1 corrects the orientation of GFP for functional expression. (b) The fluorescent reporter construct was stably integrated into HEK293T cells via lentiviral transduction and puromycin selection. The polyclonal GFP reporter cell line was then transfected with twinPE plasmid components (PE2 and four pegRNAs) and varying amounts of Bxb1 plasmid for single-transfection inversion. Cells were analyzed by flow cytometry and gated for live single cells. Quantification of GFP positive cells by flow cytometry. Values and error bars reflect the mean and s.d. of two independent biological replicates. 

single nucleofection, HEK293T cells

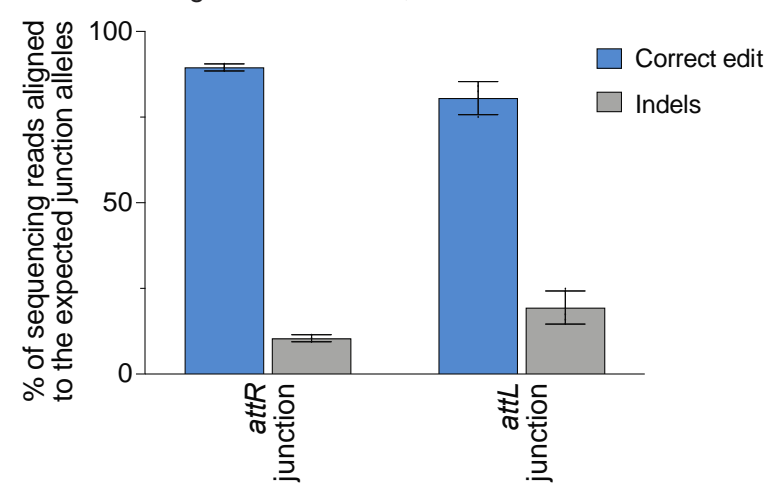

b

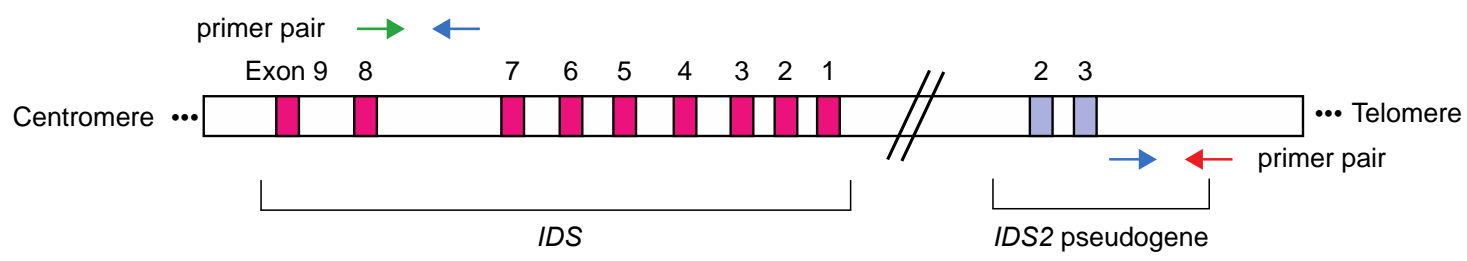

\footnotetext{
- A single primer (blue) binds to both ends of the the targeted inversion, allowing for amplification of the unedited alleles, twinPE-edited alleles, and inverted alleles.

Primer pair 1 (blue and red) can amplify the unedited alleles (402 bp), twinPE-edited alleles (336 bp), and the inverted alleles (325 bp) at junction 1 . The PCR bias during amplification at this junction was removed using UMI protocol. $\rightarrow \leftarrow \begin{aligned} & \text { Primer pair } 2 \text { (green and blue) can amplify the unedited alleles (373 bp), twinPE-edited alleles (353 bp), } \\ & \text { and the inverted alleles (347 bp) at junction } 2 .\end{aligned}$
}

\section{C}

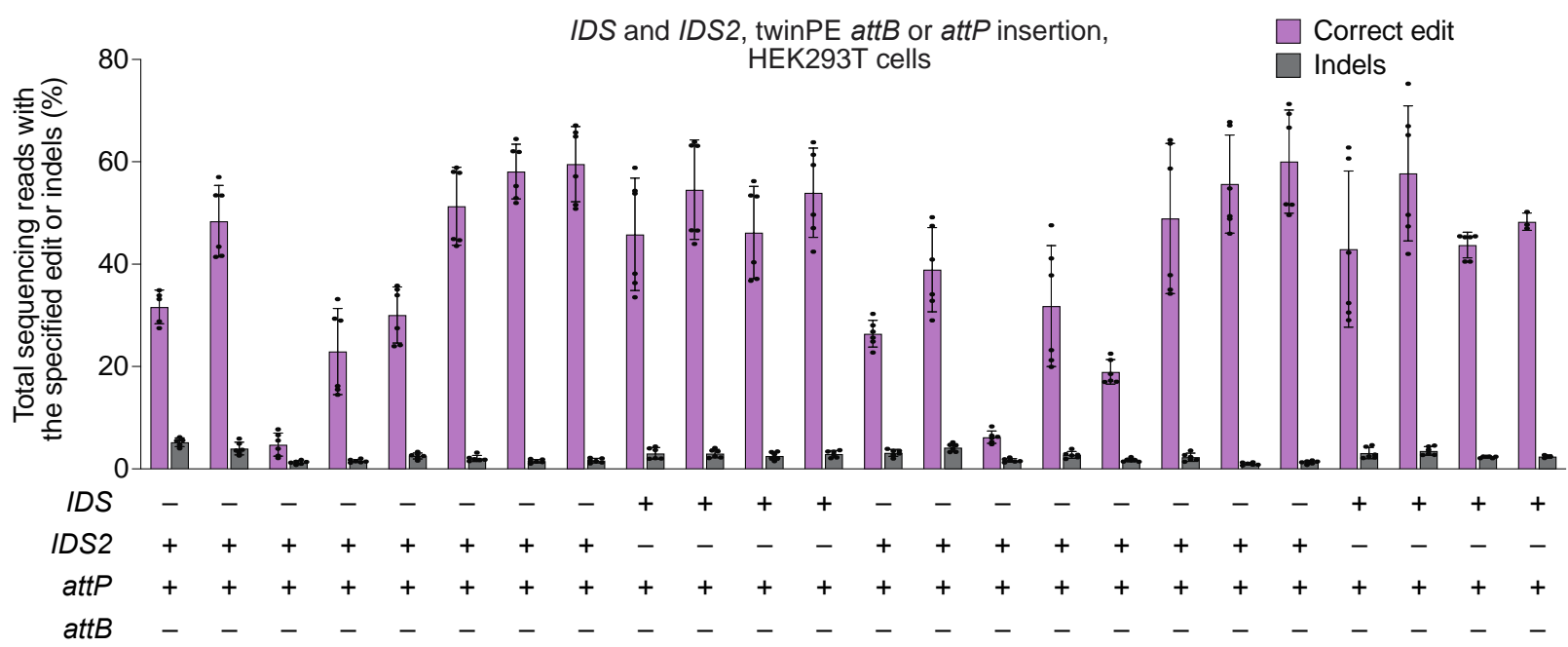

Spacer-PBS 1 A1a A1a A1c A1c A1c A1c A4b A4b C2c C2c C2c C2c A1a A1a A1c A1c A1c A1c A4b A4b C2c C2c C2c C2c Spacer-PBS 2 B2a B2a B3a B3a B4b B4b B7b B7b D1b D1b D2c D2c B2a B2a B3a B3a B4b B4b B7b B7b D1b D1b D2c D2c

$$
\begin{aligned}
& \text { attP-rev } \\
& \text { attB-fwd }
\end{aligned}
$$

$$
\begin{aligned}
& \text { attP-fwd } \\
& \text { attB-rev }
\end{aligned}
$$

Extended Data Fig. 9 | TwinPE and Bxb1 recombinase-mediated inversion between IDS and IDS2. (a) Assessment of the inverted IDS junction purity by high-throughput sequencing in HEK293T cells. Frequency of expected junction sequences containing att $R$ and attL recombination products after twinPE and BxB1-mediated single-step inversion. The product 
purities range from $81-89 \%$. Values and error bars reflect the mean and s.d. of three independent biological replicates. (b) Schematic diagram of the designed PCR strategies for quantifying IDS inversion efficiency. Primer pair 1 (green forward and blue reverse primer) can amplify the unedited alleles (403 bp), twinPE-edited alleles (337 bp), and the inverted alleles (326 bp) at junction 1 in a single PCR reaction. Due to the size difference, a UMI protocol was applied to eliminate PCR bias during quantification of inversion efficiency. Similarly, using primer pair 2 (red forward and blue reverse primer), the unedited alleles (346 bp), twinPE-edited alleles (326 bp), and inverted alleles (320 bp) at junction 2 can be amplified in a single PCR reaction. Amplicons can then be sequenced by standard highthroughput sequencing protocols for amplicon sequencing. (c) Screening of pegRNA pairs for the insertion of Bxb1 attB and attP sequences at IDS and IDS2. TwinPE editing was tested with standard pegRNAs and epegRNAs containing a 3' evoPreQ1 motif. Values and error bars reflect the mean and s.d. of three independent biological replicates. 
bioRxiv preprint doi: https://doi.org/10.1101/2021.11.01.466790; this version posted November 2. 2021. The copyright holder for this preprint (which was not certified by peer review) is the author/funder, who has granted bioRxiv a license to display the preprint in perpetuity. It is made available under aCC-BY-NC 4.0 International license.

a TwinPE-mediated attB insertion in CCR5 region 2

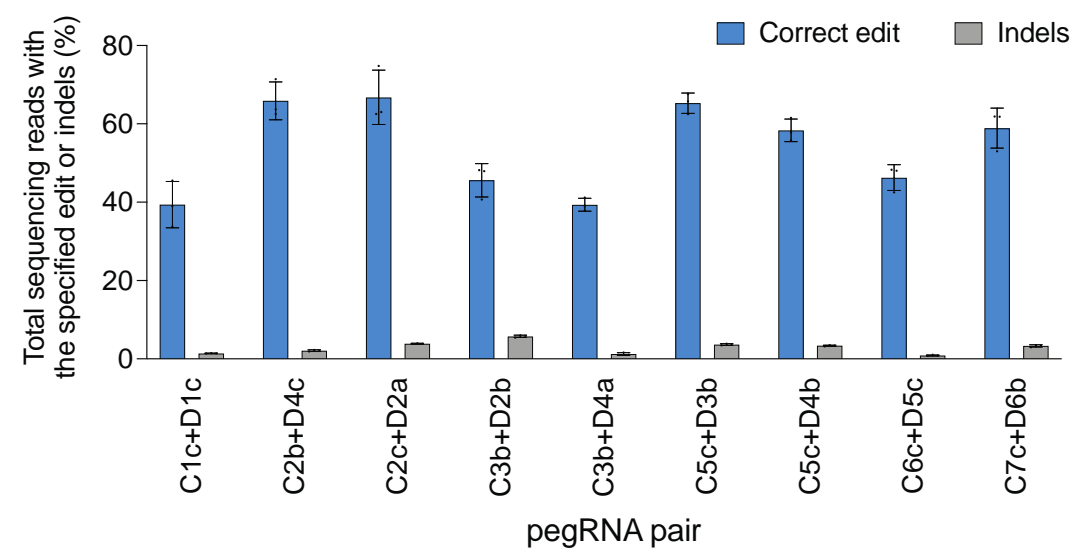

b
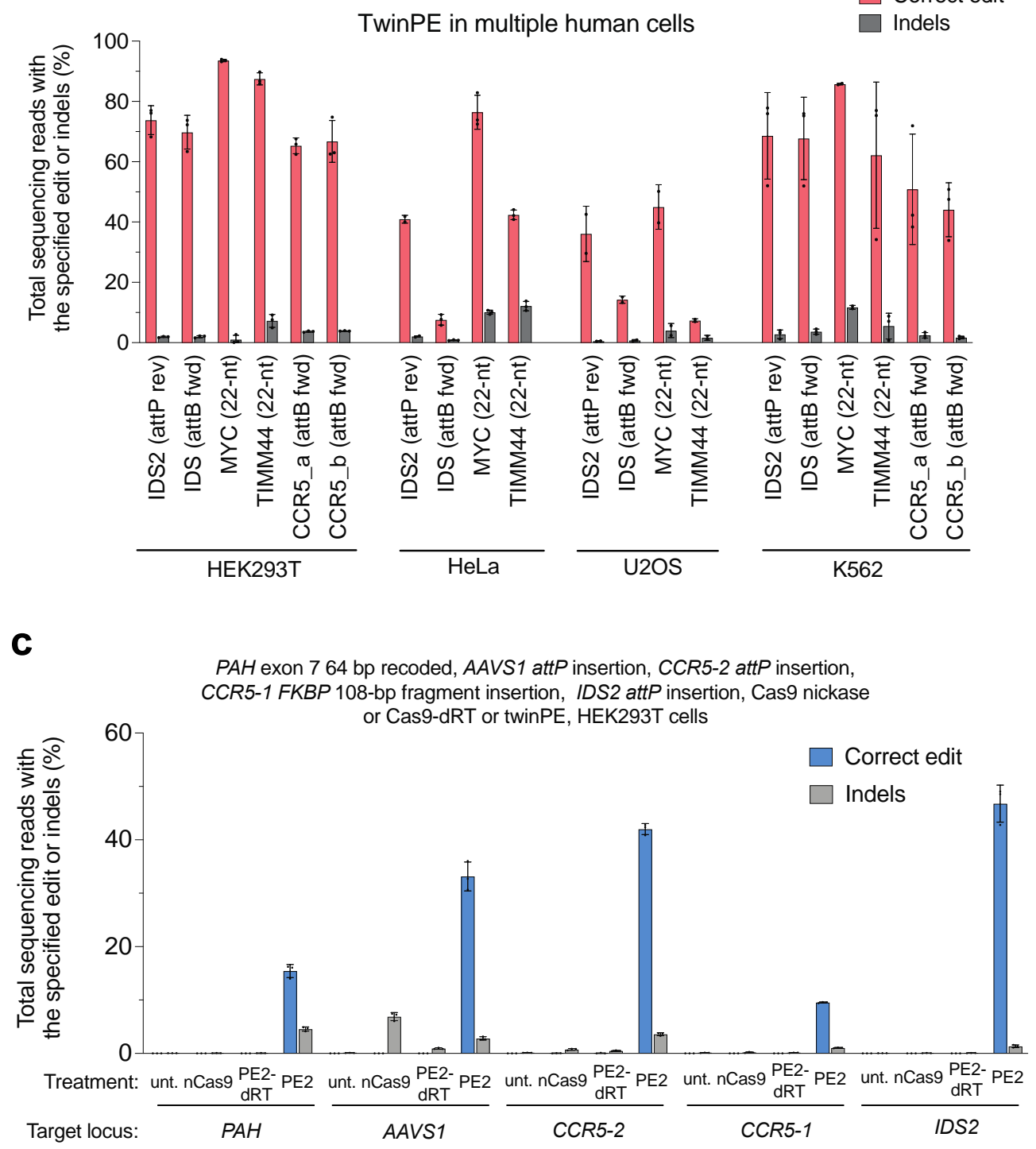

Extended Data Fig. 10 | Twin prime editing mediated insertion in CCR5 region 2 in HEK293T cells, twin prime editing in multiple human cell lines, and editing activity of 
Cas9 nickase and PE2-dead RT variants. (a) TwinPE-mediated endogenous sequence replacement with Bxb1 attB attachment site in CCR5 region 2 in HEK293T cells. (b) TwinPEmediated endogenous sequence replacement with attP, attB, or 22-nt DNA sequences in multiple human cell lines. Six different pegRNA pairs targeting five loci were tested in HEK293T, HeLa, U2OS and K562 cells. HEK293T and HeLa cell were transfected with PE2 and pegRNA plasmids via Lipofectamine 2000 (Thermo Fisher) and TransIT-HeLaMonster (Mirus), respectively. U2OS and K562 cells were nucleofected using Lonza 4D-Nucleofector and SE kit. DNA loci and the specified insertion edits are shown in the x-axis. (c) HEK293T cells were transfected with twinPE pegRNA pairs and either Cas9-H840A nickase (nCas9), PE2-dRT (a PE2 variant that contains K103L and R110S inactivating mutations to the RT domain), or PE2. Treatment with either nCas9 or PE2-dRT did not result in desired edits, while PE2 installed the specified edits as indicated. Values and error bars in (a) and (c) reflect the mean and s.d. of three independent biological replicates. Values and error bars in (b) reflect the mean and s.d. of at least two independent biological replicates. 